\title{
The late stages of the evolution of intermediate-mass primordial stars: the effects of overshooting
}

\author{
P. Gil-Pons ${ }^{1}$, J. Gutiérrez ${ }^{1}$, and E. García-Berro ${ }^{1,2}$ \\ 1 Departament de Física Aplicada, Escola Politécnica Superior de Castelldefels, Universitat Politècnica de Catalunya, \\ Avda. del Canal Olímpic s/n, 08860 Castelldefels, Spain \\ e-mail: [pilar;jordi;garcia]@fa.upc.edu \\ ${ }^{2}$ Institute for Space Studies of Catalonia, c/Gran Capità 2-4, Edif. Nexus 104, 08034 Barcelona, Spain
}

Received 19 May 2006 / Accepted 6 December 2006

ABSTRACT

\begin{abstract}
Aims. We compute and analyze the evolution of primordial stars of masses at the ZAMS between $5 M_{\odot}$ and $10 M_{\odot}$, with and without overshooting. Our main goals are to determine the nature of the remnants of massive intermediate-mass primordial stars and to check the influence of overshooting in their evolution.

Methods. Our calculations cover stellar evolution from the main sequence phase until the formation of the degenerate cores and the thermally pulsing phase.

Results. We have obtained the values for the limiting masses of Population III progenitor stars leading to carbon-oxygen and oxygenneon compact cores. Moreover, we have also obtained the limiting mass for which isolated primordial stars would lead to core-collapse supernovae after the end of the main central burning phases. Considering a moderate amount of overshooting, the mass thresholds at the ZAMS for the formation of carbon-oxygen and oxygen-neon degenerate cores shift to smaller values by about $2 M_{\odot}$. As a by-product of our calculations, we have also obtained the structure and composition profiles of the resulting compact remnants.

Conclusions. As opposed to what happens with solar metallicity objects, the final fate of primordial stars is not straightforwardly determined from the mass of the compact cores at the end of carbon burning. Instead, the small mass-loss rates typically associated with stellar winds of low metallicity stars might allow the growth of the resulting degenerate cores up to the Chandrasekhar mass, on time scales one or two orders of magnitude shorter than the time required to lose the envelope. This would lead to the formation of supernovae for initial masses as small as $\sim 5 M_{\odot}$.
\end{abstract}

Key words. stars: evolution - stars: AGB and post-AGB - stars: white dwarfs - stars: supernovae: general

\section{Introduction}

Primordial stars - that is, stars formed in the early Universe are direct inheritors of the matter synthesized during the Big Bang (Alpher \& Herman 1950; Olive 2000). The initial composition of these objects - which is characterized by the absence of metals - determines their evolution and, ultimately, the fundamental properties of the resulting stellar remnants, as well as the amount and composition of the matter returned to the interstellar medium after the main evolutionary phases. Therefore, the evolution of these stars is important for a correct modeling of the population of the degenerate remnants of Population III stars and, hence, for a better understanding of the distribution of baryonic dark matter, whereas the significance of the ejected material rests on its influence on Galactic chemical evolution.

Even though the detection of primordial composition objects - that is, stars with $[\mathrm{Fe} / \mathrm{H}] \lesssim-8.3$ - has not been possible up to now, during the last few years several encouraging observations have been made. For instance, Bessel \& Norris (1984) detected a red giant with $[\mathrm{Fe} / \mathrm{H}] \approx-4.6$ and Christlieb et al. (2002) measured the metallicity of HE 0107-5240, a low mass star with $[\mathrm{Fe} / \mathrm{H}] \approx-5.3$. Even more recently, Frebel et al. (2005) have observed HE 1327-2326, an unevolved low-mass object, which was found to have $[\mathrm{Fe} / \mathrm{H}] \approx-5.4$. Consequently, both the intrinsic theoretical interest and the growing observational evidence for the existence of primordial stars make the study of these objects one of the hottest topics in stellar astrophysics nowadays.

The exact shape of the initial mass function of Population III stars is far from being known. The standard view advocates for a strong bias toward very massive stars, say between 100 and $600 M_{\odot}$ - see, for example, Bromm et al. (2002). Nevertheless, arguments have been put forth recently that point to the possible existence of a population of very metal poor stars of low- and intermediate-mass. For instance, from the theoretical side, Nakamura \& Umemura $(1999,2001)$ have simulated the growth of instabilities in protostellar primordial clouds in 2 and 3 dimensions. Their results indicate that the initial mass function could be bi-modal with a peak at $\sim 100 M_{\odot}$ and a secondary peak at a few solar masses. Additionally, Machida et al. (2005) have shown that the explosion of primordial supernovae can drive the Jeans masses of primordial clouds from a few $10^{6} M_{\odot}$ to values well below $1 M_{\odot}$, hence allowing for the formation of intermediate-mass stars, but with non-negligible metallicities (of the order of $[\mathrm{Fe} / \mathrm{H}] \approx-3.0$ ). Furthermore, Johnson $\&$ Bromm (2006) have recently raised more theoretical considerations that suggest that the formation of primordial low- and intermediate-mass stars is viable. On the other hand, and from the observational side, Schneider et al. (2006), on the basis of three tentative NICMOS UDFs sources at $z \approx 10$ and the derived WMAP electron scattering optical depth, predict a rather 
standard form of the IMF, with stars within the $1 M_{\odot} \lesssim M \lessgtr$ $100 M_{\odot}$ range.

The evolution of zero metallicity stars has been analyzed in several recent publications. Just to mention some of them, and without trying to be complete, it is important to realize that Heger et al. (2001) and Heger \& Woosley (2000) have focused on massive objects, whereas Limongi et al. (2000), Marigo et al. (2001), Chieffi et al. (2001), and Siess et al. (2002) have thoroughly studied the evolution of low- and intermediate-mass stars. However, the evolution of massive intermediate-mass primordial stars has been largely ignored, except for the case of the evolutionary sequence of a $9 M_{\odot}$ star presented in Gil-Pons et al. (2005). One of the reasons for this is probably the heavy computational load involved in computing the evolution during the carbon burning phase for this mass range (Ritossa et al. 1995; García-Berro et al. 1997; Iben et al. 1997; Ritossa et al. 1999). This is in sharp contrast with the situation for stars of Population I and II, which have been thoroughly analyzed. For instance, the mass thresholds for objects that lead to different types of remnants have been determined for Population I and II stars by Heger et al. (2003), as well as by Eldridge \& Tout (2004), who computed a rich grid of stellar models ranging both in mass - from 5 to $200 M_{\odot}$ - and metallicity - from $Z=10^{-5}$ up to $Z=0.05$. The latter authors also paid special attention to the adopted mass-loss rates, including their variation with the metallicity, and to the effects of overshooting. Finally, it is worth mentioning as well that the influence of Population III stars on the formation and ejection of isotopes in the intergalactic medium has been extensively studied by Goriely \& Siess (2002) and by Abia et al. (2001).

In this paper, we describe the final stages of the evolution of massive intermediate mass primordial stars. Reliable results on this matter are essential inputs for Galactic chemical evolution models, as well as for the supernova theory. Particularly, thermonuclear supernovae (SNeIa), in which a carbon-oxygen white dwarf overcomes the Chandrasekhar limit, must rely on accurate initial-to-final mass relations to reproduce the observed supernova rate, and its dependence on metallicity could represent a source of diversity on classical SNeIa at different redshifts. Specifically, we outline the evolution of the progenitor stars and determine the mass thresholds to obtain carbon-oxygen (CO), oxygen-neon $(\mathrm{ONe})$ degenerate cores and core collapse supernovae at the end of the main central burning stages. The stars hosting $\mathrm{CO}$ degenerate cores could give rise to $\mathrm{CO}$ novae or to SNeIa if belonging to a close binary system; those hosting ONe degenerate cores could also form ONe novae or supernovae by the accretion-induced collapse mechanism (Canal \& Schatzman 1976; Gutiérrez et al. 1996, 2005), and core-collapse supernovae would directly form from isolated stars when nuclear burning proceeds all the way to the formation of an iron peak element dominated core.

An important part of our results consist of justifying that the formation of supernovae does not require ZAMS masses as high as in the case of solar metallicity objects but, instead, $6 M_{\odot}$ stars (and probably even less massive ones) are enough to produce a supernova explosion. This possibility had already been suggested, for instance, by Arnett (1969), by Iben \& Renzini (1983), by Willson (2000), and, more recently, by Zijlstra (2004). The reason for such peculiar behavior has to do with the characteristics of stellar winds in primordial stars. Low metallicity stars are supposed to drive weaker stellar winds (Bowen \& Willson 1991; Willson 2000) than their solar metallicity counterparts. However, some caution is mandatory here, as the cited works are theoretical with phenomenological character, and observations do not fully support - or discard - their findings yet. Therefore, the degenerate cores are able to grow significantly due to shell burning before the envelope is lost. As we will show below, the cores resulting from the evolution of massive intermediate-mass primordial stars will be able to grow up to the Chandrasekhar mass and, therefore, undergo a supernova explosion.

Of course, all these results depend sensitively on the adopted prescription for convection and, in particular, on the degree of overshooting. Overshooting represents one of the main unknowns in stellar evolution, as observations indicate more mixing than predicted by standard, local, and non-local convective prescriptions. Rotation and purely three-dimensional hydrodynamic phenomena could explain this extra mixing, but this is still the subject of an active, ongoing debate. Hence, we have performed calculations both with and without overshooting to clarify its effects on the initial-to-final mass relation. Previous works on overshooting - see, for example, Young et al. (2003) and Demarque et al. (2004) for recent, enlightening studies - indicate that overshooting produces an increase in the mass of the nuclear exhausted core, a change of its internal composition, and a modified chemical composition of the surface layers. All these effects are relevant for the purposes of our work. In particular, the extra mixing in the surface layers is of the maximum importance, as Population III stars do not experience a significant mass-loss due to the small opacity of their outer layers. The extra mixing due to overshooting could increase the metallicity of the outer layers and, consequently, increase the mass-loss rates, thus changing the final mass of the remnants.

The paper is organized as follows. In Sect. 2 we describe the overall evolution prior to carbon burning. Section 3 is devoted to studying the different carbon burning episodes in depth. In Sect. 4 we comment on the thermally pulsing phase for this mass range, whereas in Sect. 5 we describe the properties of the cores of these stars. Finally, in Sect. 6 we elaborate on the possible evolutionary outcomes that can be envisaged for these stars, discussing in depth the effects of the adopted mass-loss rates, whilst in Sect. 7 we summarize our major findings, we draw our conclusions, and we discuss their significance.

\section{Overall evolution prior to carbon burning}

We have followed the evolution of zero-metallicity models with masses at the ZAMS between 5 and $10 M_{\odot}$, and initial values for hydrogen and helium abundances of $X=0.77$ and $Y=0.23$, respectively. Our calculations have been performed both taking into account and neglecting overshooting. In doing so, we have used the evolutionary code previously described in Gil-Pons et al. (2005), which is, itself, an update of the code described in detail in Ritossa et al. (1995). We remark that the opacities are obtained by interpolating in the tables of Iglesias \& Rogers (1993). The only novelty with respect to the previous implementations of our code is that for the calculations reported here we have incorporated overshooting. This will allow us to isolate the differences introduced by the treatment of convective boundaries in the evolutionary calculations presented here. Specifically, the prescription used for the calculation of the convective edges is the one described in Eldridge \& Tout (2004), in which the standard Schwarzschild criterion is modified to let convection be the main energy transport mechanism in those zones of the star where $\nabla_{\text {rad }}>\nabla_{\text {ad }}-\delta$, with

$\delta=\frac{\delta_{\mathrm{OV}}}{2.5+20 \zeta+16 \zeta^{2}}$ 
Table 1. Main nuclear burning times and core masses.

\begin{tabular}{ccccccccc}
\hline \hline \multirow{2}{*}{$M_{\text {ZAMS }} / M_{\odot}$} & \multicolumn{2}{c}{$t_{\mathrm{CHB}}\left(\times 10^{14} \mathrm{~s}\right)$} & \multicolumn{2}{c}{$M_{\mathrm{He}} / M_{\odot}$} & \multicolumn{2}{c}{$t_{\mathrm{CHeB}}\left(\times 10^{14} \mathrm{~s}\right)$} & \multicolumn{2}{c}{$M_{\mathrm{CO}} / M_{\odot}$} \\
\cline { 2 - 9 } & no OV & OV & no OV & OV & no OV & OV & no OV & OV \\
\hline 5.0 & 19.8838 & 21.2417 & 1.17 & 1.32 & 23.6791 & 24.2174 & 0.64 & 0.80 \\
6.0 & 13.8396 & 15.3209 & 1.18 & 1.60 & 16.2184 & 17.0675 & 0.65 & 0.90 \\
7.0 & 10.5091 & 11.8733 & 1.20 & 2.23 & 12.1470 & 12.8494 & 0.83 & 1.15 \\
8.0 & 8.3796 & 9.4119 & 1.48 & 2.30 & 9.7551 & 10.3818 & 1.09 & 1.38 \\
9.0 & 6.8994 & 7.7360 & 1.85 & 2.35 & 8.0519 & 8.5778 & 1.18 & 1.50 \\
10.0 & 6.0116 & 6.7436 & 2.09 & 2.38 & 6.8359 & 7.3791 & 1.30 & 1.76 \\
\hline
\end{tabular}

where $\zeta=P_{\text {rad }} / P_{\text {gas }}$ and $\delta_{\text {OV }}$ is set to be equal to 0.12 (Schröder et al. 1997).

The evolution prior to carbon burning follows the main trends previously described in Gil-Pons et al. (2005) very closely. That is, core hydrogen burning (CHB) begins through the pp-chains until small amounts of carbon $\left(X(\mathrm{C})=10^{-10}\right.$ by mass) form and allow the onset of the CNO cycle. The rest of the CHB phase therefore proceeds at the higher central temperatures that characterize this cycle. Once CHB has ended, core contraction proceeds and the conditions for core helium burning are reached in the central regions of the star. Those for hydrogen burning are attained at the layers just above the hydrogen exhausted core. Contrary to what happens with solar metallicity stars, all these phases take place at the blue region of the Hertzsprung-Russell diagram. Furthermore, a hydrogen shell burning in a metal-free environment is not able to produce the overall expansion of the stellar envelope that characterizes the red giant branch.

As mentioned in Gil-Pons et al. (2005), the main differences between the evolutionary tracks for $Z=0$ and those of $Z=Z_{\odot}$ are the longer time spent during the main sequence phase, the higher surface luminosities reached during $\mathrm{CHB}$, and the larger helium-exhausted core of the $Z=0$ models. Table 1 shows the duration of the CHB phase $\left(t_{\mathrm{CHB}}\right)$, the mass of the resulting helium core $\left(M_{\mathrm{He}}\right)$, the time at which carbon burning begins $\left(t_{\mathrm{CB}}\right)$, and the mass of the corresponding carbon-oxygen core. As can be seen, the calculations with overshooting yield time scales for core hydrogen burning that are about a $12 \%$ longer when compared to the cases computed without taking into account overshooting. The helium cores after the CHB phase has been completed are also about $50 \%$ larger and this is the reason why the core helium burning $(\mathrm{CHeB})$ phase lasts about $6 \%$ longer in the cases in which overshooting was incorporated. Finally, the masses of the $\mathrm{CO}$ cores $\left(M_{\mathrm{CO}}\right)$ prior to carbon burning are considerably larger as well, that is, about $25 \%$ more massive for the cases computed with overshooting.

Figure 1 shows the evolution of the central temperature versus the central density for both sets of models. The cases in which overshooting was disregarded and the cases in which overshooting was taken into account are represented differently. For the sake of conciseness we only show the evolutionary sequences of the 7,8 , and $10 M_{\odot}$ model stars. As can be seen, only small differences appear between both sets of models during the CHB phase. However, the core contraction phases that follow the $\mathrm{CHB}$ and the $\mathrm{CHeB}$ phases take place at higher temperatures and reach lower values for the maximum central density in the cases computed with overshooting.

The evolution in the Hertzsprung-Russell diagram of the same model stars is shown in Fig. 2. In the upper panel the track of the $7 M_{\odot}$ star is shown, and in the middle and lower panel we represent the evolution of the $8 M_{\odot}$ and $10 M_{\odot}$ models, respectively. The cases with no overshooting are represented, as are

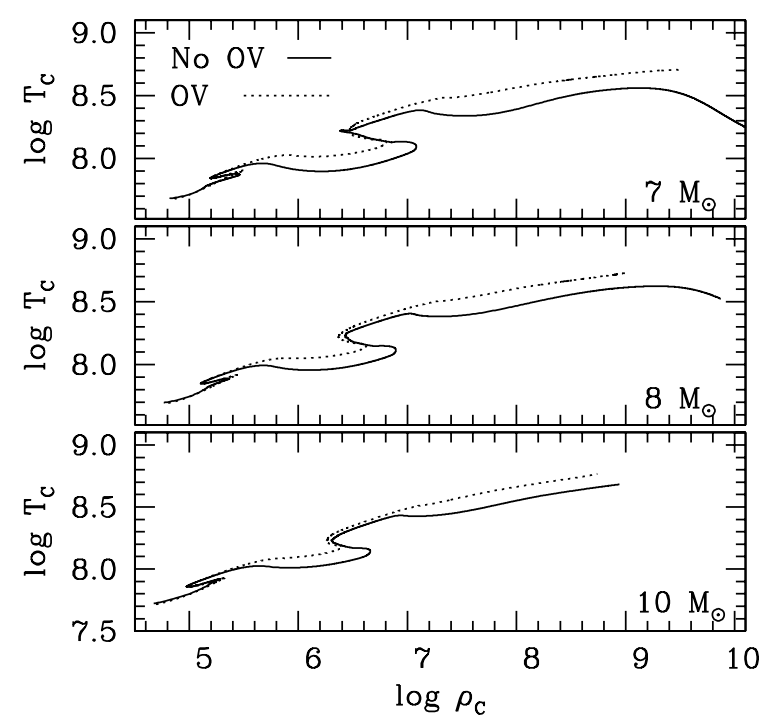

Fig. 1. Evolution in the $\log \rho_{\mathrm{c}}-\log T_{\mathrm{c}}$ plane of our model stars up to the point where carbon is ignited. The solid lines correspond to the case in which no overshooting was taken into account, whereas the dotted lines correspond to the calculations in which overshooting was incorporated.

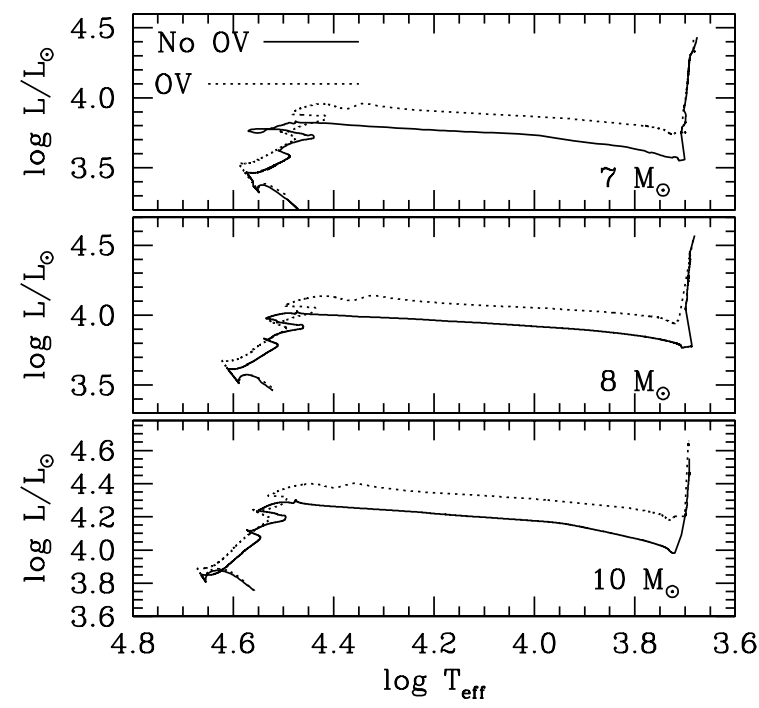

Fig. 2. Evolutionary tracks in the Hertzsprung-Russell diagram of our model stars. The solid lines correspond to the case in which overshooting was disregarded, whereas the dotted lines show the evolution for the case in which overshooting was due taken into account.

the cases with overshooting. As can be seen, CHB takes place at higher effective temperatures for the models computed with overshooting, and the Hertzsprung gap occurs at larger luminosities. In summary, the models computed with overshooting 


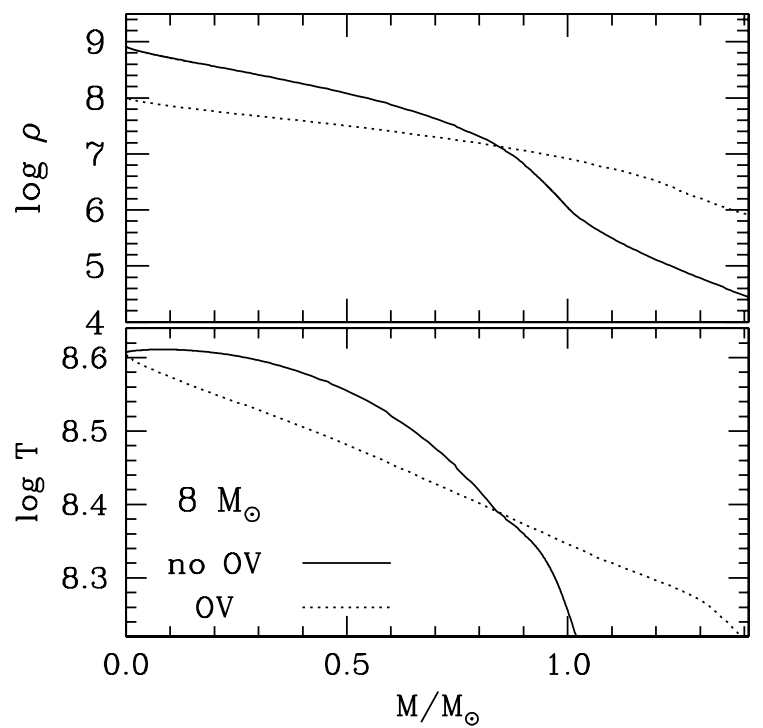

Fig. 3. Upper panel: density profile of the degenerate carbon-oxygen cores for the $8 M_{\odot}$ model star just before $\mathrm{C}$ ignition for the case computed without overshooting (solid line) and with overshooting (dotted line). Lower panel: temperature profiles of the carbon-oxygen cores computed without overshooting (solid line) and with overshooting (dotted line) for the same model star.

behave as if they were more massive than their counterparts computed without overshooting.

This can be better seen by comparing the masses of the $\mathrm{CO}$ cores just before the carbon burning phase (Table 1). Also, the density and temperature profiles just before carbon ignition - which are shown in Fig. 3 for the $8 M_{\odot}$ model star - help in displaying this distinctive behavior. Particularly, the model computed with overshooting presents a smaller central density and a shallower density profile - see the top panel of Fig. 3 - leading to a larger region of high densities and, therefore, to a larger core than the model computed without taking into account overshooting. Note, however, that the central temperatures (see the bottom panel of Fig. 3) of both models are remarkably similar, although the mass of the core is rather different. Figure 4 shows the chemical profiles of the same model star. The model computed with no overshooting and the model computed when due account is taken of overshooting are represented differently. The former appears to be $0.3 M_{\odot}$ larger than the latter, but the mass abundances of carbon and oxygen in the central regions of the star are practically the same in both cases. All these quantities are particularly important because, as it will be shown in Sect. 4 below, the development of extensive carbon burning and, ultimately, the formation of an oxygen-neon white dwarf crucially depend on them. The mass threshold at the ZAMS for the formation of supernovae from single $Z=0$ stars also depends significantly on the adopted prescription for determining the edge of the convective cores.

\section{The carbon-burning phase}

Figure 5 represents the temporal evolution of the base of the convective envelope (BCE) as well as the luminosities associated with carbon burning and helium burning for the $8 M_{\odot}$ model stars computed without overshooting (top panel) and with overshooting (bottom panel), which is a representative case. The convective regions associated with carbon burning are also shown for both models. We remark at this point that neither the $6 M_{\odot}$ nor

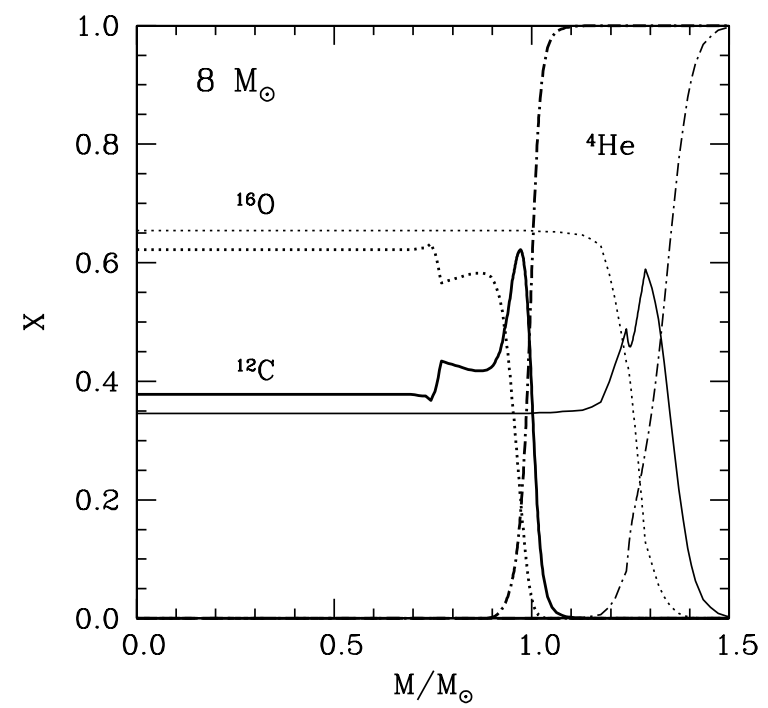

Fig. 4. Composition profiles of the degenerate $\mathrm{CO}$ cores of the $8 M_{\odot}$ model star computed with overshooting (thin lines) and disregarding overshooting (thick lines) just before carbon burning sets in.

the $7 M_{\odot}$ model stars computed without overshooting develop carbon burning extensively - although for the latter case there is partial carbon burning, reaching the carbon luminosity a maximum value of only $L / L_{\odot} \simeq 10-$ and, therefore, the remnant cores are composed mainly of carbon and oxygen. In fact, our calculations show that the mass threshold at the ZAMS for effective carbon burning in the partially degenerate core to obtain an ONe remnant is $\approx 7.8 M_{\odot}$, when no overshooting is considered. This mass threshold is $\approx 6.0 M_{\odot}$ when overshooting is taken into account.

The first important feature to be noticed in Fig. 5 is the fast advance of the base of the convective envelope that, for both models, occurs shortly after the end of the core helium burning phase and the onset of helium burning in a shell - at $t \simeq 9.757 \times 10^{14} \mathrm{~s}$ for the model computed without overshooting and at $t \simeq 10.2907 \times 10^{14} \mathrm{~s}$ for the model computed with overshooting. This first advance inward, the so-called "second" dredge-up episode - although, in fact, it is the first dredge-up episode (Gil-Pons et al. 2005) - is followed by a very slow penetration of the BCE that finally allows for a moderate enrichment in metals of the hydrogen-rich envelope. Although most of the material dredged-up consists of helium, which does not contribute to the metal content of the convective envelope, some small amounts of metals are indeed dredged-up. The metallicity of the envelope depends sensitively on the mass of the star. For instance, the metallicity of the envelope of the $5 M_{\odot}$ model star computed without overshooting is $Z_{\mathrm{env}} \approx 10^{-9}$, whereas for the $9 M_{\odot}$ star we obtain $Z_{\text {env }} \approx 10^{-4}$. The models computed with overshooting show the same behavior: $Z_{\mathrm{env}} \approx 10^{-7}$ for the $5 M_{\odot}$ star and $Z_{\text {env }} \approx 10^{-3}$ for the $8 M_{\odot}$. As it will be shown below, this enrichment plays a key role in the final fate of massive intermediate-mass primordial stars. The reason for this behavior can be understood by studying the evolution of the $8 M_{\odot}$ model star computed without overshooting, which shows a particular feature associated with the inner advance of the convective envelope. For this model the first carbon flash at $t \sim 9.766 \times 10^{14} \mathrm{~s}$ occurs near the center of the star, but the associated convective zone extends to layers relatively close to the BCE (at $M_{r} \simeq 0.9 M_{\odot}$ ) and, thus, allows for a decrease of the degeneracy parameter of the outermost layers of the core. Consequently, the density and 


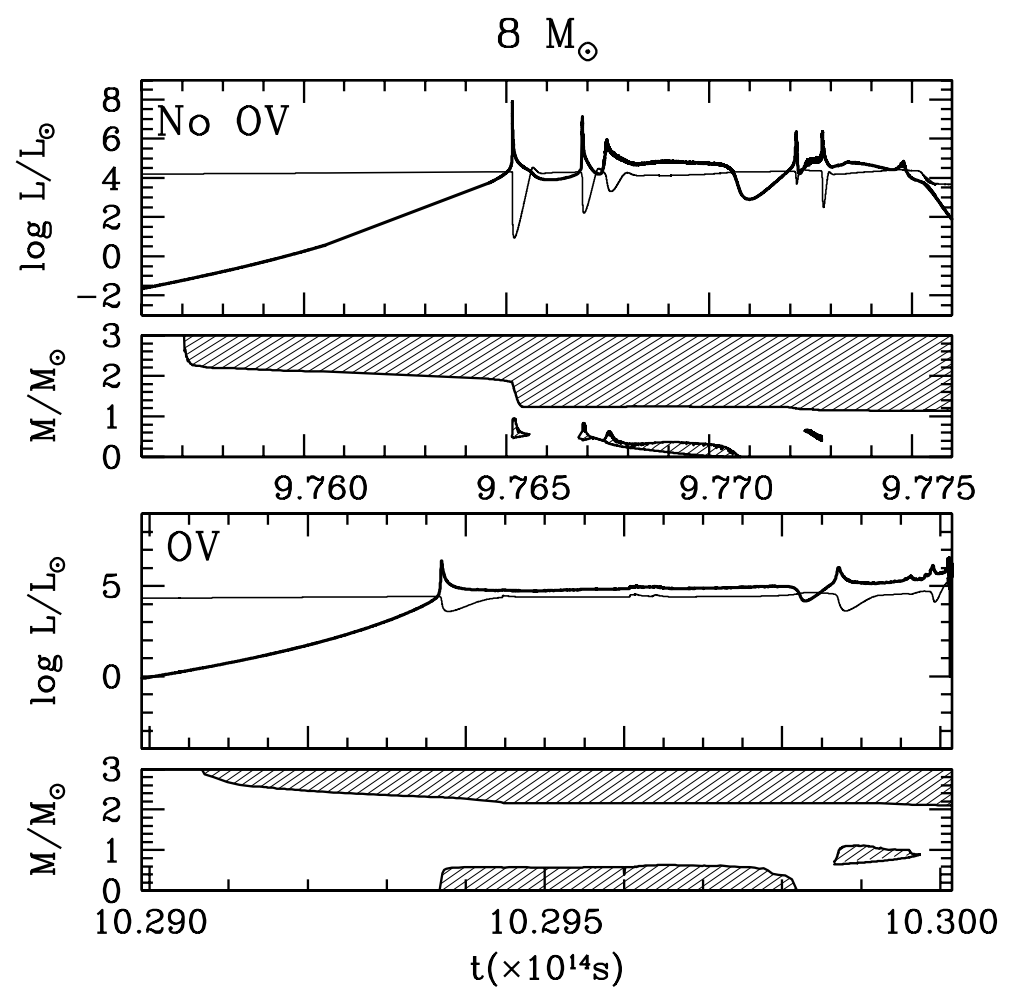

Fig. 5. Temporal evolution of the main structural parameters during the bulk of the carbon burning phase for the $8 M_{\odot}$ model computed without overshooting (upper panel) and with it (bottom panel). The shaded areas represent the evolution of the convective regions, the thick solid line depicts the evolution of the carbon burning luminosity, and the thin solid line shows the helium burning luminosity.

temperature barriers decrease and this results in an additional advance of the BCE. This, in turn, causes an enrichment in metals of the convective envelope. For the model computed with overshooting this feature is absent, since carbon ignition occurs at the center, too far from the edge of the carbon-oxygen partially degenerate core (which is located at $M_{\mathrm{BCE}} \simeq 2.12 M_{\odot}$ ) to have any relevant consequence on the behavior of the convective envelope.

The $8 M_{\odot}$ model computed without overshooting actually undergoes 5 significant carbon flashes, the first of which is the most powerful one $\left(\sim 10^{8} L_{\odot}\right)$. Once the overall degeneracy of the core decreases after this flash, the following ones proceed with more moderate luminosities, as usually occurs for solar metallicity models of equivalent masses during this phase. As also occurs in solar metallicity stars of the same mass, each thermonuclear flash generates an associated convective shell and an abrupt decrease of the He luminosity (see Fig. 5). This is a consequence of the fast injection of energy in the uppermost layers of the partially degenerate core, which consequently expand and cool and, therefore, effectively switch-off the He burning shell. For the model computed taking overshooting into account, carbon ignition occurs at the center. This is a consequence of the smaller central densities and lower temperatures achieved before carbon ignition (see Fig. 3), which lead respectively to a smaller degeneracy parameter and to smaller neutrino leaks. Consequently, the temperature profile shows a central maximum, in contrast with the situation for the $8 M_{\odot}$ model computed without overshooting, for which the maximum temperature occurs relatively far from the center. Additionally, we do not obtain as many flashes as for the model in which overshooting was not taken into account. In fact, the first carbon flash has a smaller strength $\left(L_{\mathrm{C}} \simeq 10^{7} L_{\odot}\right)$. This also stems from the fact that the degeneracy in the core is substantially smaller. After the sudden injection of nuclear energy, the core of this model star expands and the overall degeneracy decreases, leading to a more gentle carbon burning phase, in which carbon is burnt in almost stationary conditions. Moreover, since the outer edge of the central convective carbon burning region is located deep in the core, the effect on the $\mathrm{He}$ burning shell is substantially smaller and, consequently, the $\mathrm{He}$ burning luminosity decreases less than in the previous case.

\section{The thermally pulsing phase}

For the models that fail to ignite carbon under partially degenerate conditions, a thermally pulsing phase ensues soon after helium burning ceases in the central regions. For these models the helium-burning shell is very close to the base of the hydrogenrich convective envelope. For instance, for the $7 M_{\odot}$ model sequence computed without overshooting, the helium-burning shell is only $\sim 4.1 \times 10^{-4} M_{\odot}$ away from the H-He discontinuity. Consequently, the temperature at this discontinuity increases to the extent that hydrogen-burning is reactivated and the thermally pulsing AGB phase (TP-AGB) phase ensues shortly afterward. For the models that do ignite carbon, the thermally pulsing Super-AGB (TP-SAGB) phase ensues after the carbon-burning phase has been completed. This, for instance, is the case of the $8 M_{\odot}$ model star computed without overshooting. Again, in this case the helium-burning shell (located at $M_{r} \simeq 1.049875 M_{\odot}$ ) is very close to the $\mathrm{H}-\mathrm{He}$ discontinuity (located at a mass coordinate $M_{r} \simeq 1.08169596 M_{\odot}$ ) and the hydrogen burning shell resurrects as well. Note, however, that Yoon et al. (2004) have recently shown that other parameters, like rotation, define the stability of shell burning.

In Fig. 6 we display the helium luminosity for the first few thermal pulses of some selected models. Note that although the time scales are rather different, the amplitudes of the pulses are 

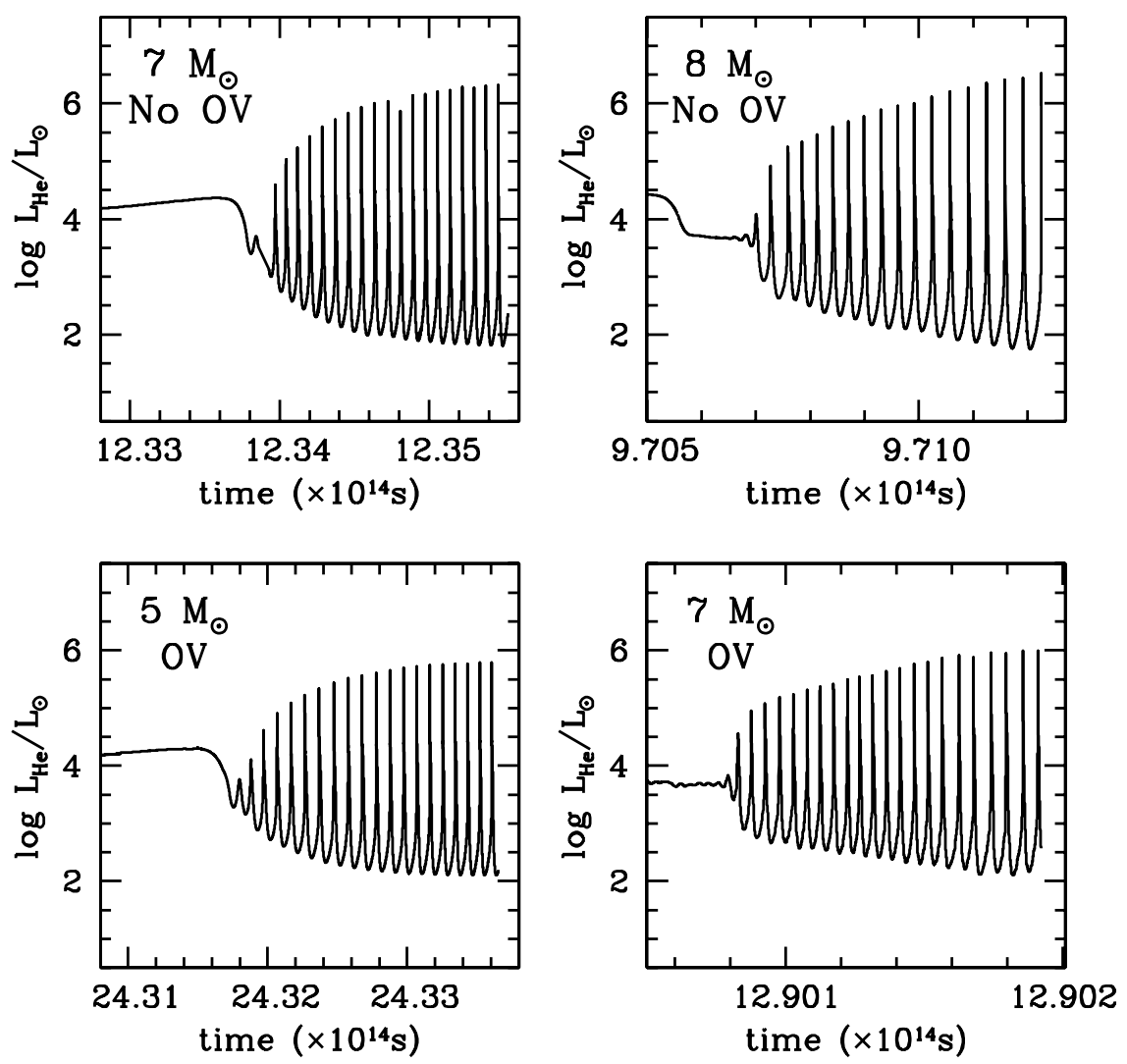

Fig. 6. Temporal evolution of $L_{\mathrm{He}}$ during the early TP-(S)AGB phase of the $7 M_{\odot}$ model computed without overshooting (upper left panel), the $8 M_{\odot}$ without overshooting (upper right panel), the $5 M_{\odot}$ model computed with overshooting (lower left panel), and the $7 M_{\odot}$ with overshooting (lower right panel).

similar. The fact that these stars develop the TP-(S)AGB phase in a way similar to that of solar metallicity models of analogous masses is related to the dredge-up processes that follow the development of shell helium burning. The associated metal enrichment of the base of the hydrogen-rich envelope, as well as its heating due to the proximity to the helium burning shell (HeBS), allow, as we have just explained, for a new onset of hydrogenburning through the CNO cycle, which occurs as soon as the distance between the HeBS and the base of the hydrogen-rich envelope is about $10^{-4} M_{\odot}$. From this time on, hydrogen and helium burning alternate themselves as main energy suppliers. In the next two subsections we give some details about the thermal pulses that ensue in stars that have developed either a carbonoxygen or an oxygen-neon core.

\subsection{Models with carbon-oxygen cores}

To keep consistency with the existing literature, we will refer to these models as TP-AGB models. The upper and lower left panels of Fig. 6 show, respectively, the first few thermal pulses of the $7 M_{\odot}$ model computed without overshooting and those corresponding to the $5 M_{\odot}$ model star computed with overshooting. These are representative examples of massive intermediate-mass primordial stars with carbon-oxygen cores. We recall that models with masses at the ZAMS smaller than $7.8 M_{\odot}$ never ignite carbon if no overshooting is adopted, whereas if overshooting is taken into account the mass limit is about $6 M_{\odot}$. Since both models fail to ignite carbon, the TP-AGB phase starts at the end of the $\mathrm{CHeB}$ phase. However, it is worth mentioning that before reaching the TP-AGB phase, both models have experienced a brief (and weak) carbon-burning phase, with associated luminosities that never exceed $\sim 10 L_{\odot}$. This phase is, in any case, unable to substantially modify the chemical profile of the corresponding cores. As it happens for solar metallicity TP-AGB models, each helium flash is accompanied by the expansion and subsequent cooling of the neighboring upper layers and, therefore, by the switch-off of the hydrogen burning shell. The interpulse period for the $7 M_{\odot}$ model computed without overshooting amounts to $3200 \mathrm{yr}$, whereas it is reduced to $2700 \mathrm{yr}$ for the $5 M_{\odot}$ model computed with overshooting. As it can be seen, the average values of the helium luminosity are very similar $\left(L_{\mathrm{He}} \approx 10^{4} L_{\odot}\right)$ for both models, but the maximum helium luminosity is somehow larger for the $7 M_{\odot}$ model computed without overshooting.

The upper left panel of Fig. 7 represents the evolution of the $\mathrm{BCE}$ and the pulse-driven convective zones associated with the 8th and the 9th helium flashes of the $7 M_{\odot}$ computed without overshooting, occurring at times $t \simeq 12.3446 \times 10^{14} \mathrm{~s}$ and $t \simeq 12.3455 \times 10^{14} \mathrm{~s}$, respectively. It can be seen that the fast injection of energy associated with each helium flash causes the development of an inner convective zone, as it occurs in the case of solar metallicity model stars. Simultaneously, the expansion and cooling that occur in the neighboring layers produce the extinction of the hydrogen burning shell and, as a consequence, a fast advance inwards of the BCE. By the end of the helium flash, the H-burning shell moves outward, increasing the mass of the He buffer and a new He flash occurs once enough matter is accumulated. However, the penetration of the BCE into the regions that have been processed by previous pulse-driven convective zones is hardly noticeable. Hence, the mixing of the material of the hydrogen-exhausted core with that of the convective envelope does not allow for a significant variation of the 

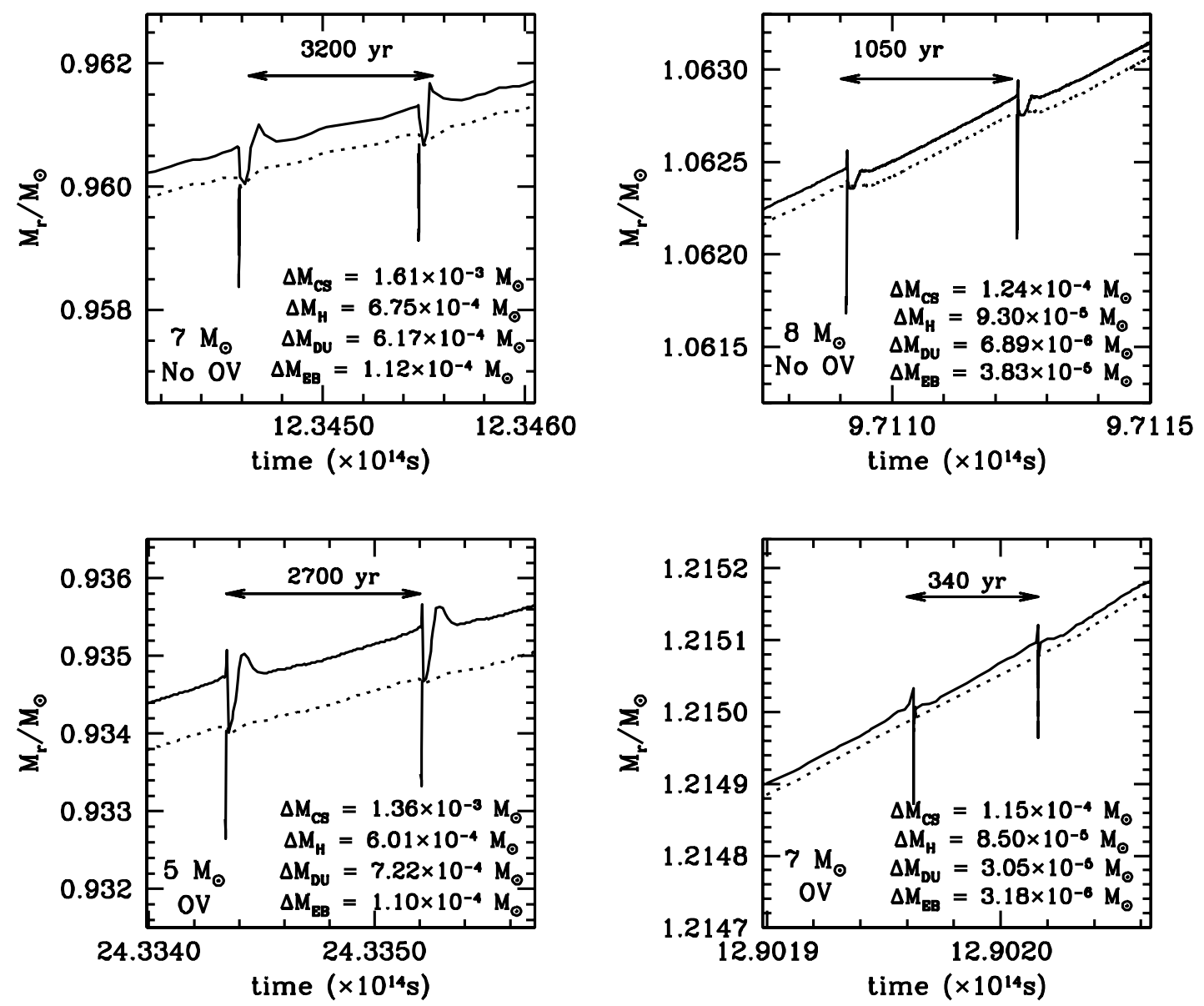

Fig. 7. Temporal evolution of the edges of the convective shell and of the base of the convective envelope during the 8th and the 9th pulses of the $7 M_{\odot}$ model star computed without overshooting (upper left panel), the 14 th and the 15 th pulses of the $8 M_{\odot}$ star computed without overshooting (upper right panel), the 18th and the 19th pulses of the $5 M_{\odot}$ model computed with overshooting (lower left panel), and the 23th and 24th pulses of the $7 M_{\odot}$ computed with overshooting (lower right panel). In the figure are given the values of $\Delta M_{\mathrm{CS}}$, the maximum mass of the convective shell; $\Delta M_{\mathrm{H}}$, the mass through which the hydrogen profile moves between pulses; $\Delta M_{\mathrm{DU}}$, the amount of mass dredged-up during pulse power-down; and $\Delta M_{\mathrm{EB}}$, the mass of the outer edge of the convective shell and the base of the convective envelope. The mass of the hydrogen-exhausted core is shown as a dotted line.

metallicity of the envelope and does not affect the subsequent evolution. In fact, the increase in metallicity that this model star experiences during each helium flash is only $\Delta Z_{\mathrm{env}} \approx 10^{-8}$. For the sake of completeness, in Fig. 7 we have included the values of the maximum mass of the convective shell during the 9 th pulse $\left(\Delta M_{\mathrm{CS}}=1.61 \times 10^{-3} M_{\odot}\right)$, the mass through which the BCE moves between pulses $\left(\Delta M_{\mathrm{H}}=6.75 \times 10^{-4} M_{\odot}\right)$, the mass dredged-up after the $9^{\text {th }}$ helium flash $\left(\Delta M_{\mathrm{DU}}=6.17 \times 10^{-4} M_{\odot}\right)$, and the thickness of the region between the outermost edge of the convective shell and the base of the convective envelope $\left(\Delta M_{\mathrm{EB}}=1.12 \times 10^{-4} M_{\odot}\right)$.

To this regard it is important to mention here that the efficiency of the third dredge-up strongly depends, not only on the treatment of convection, but also on the numerical treatment. Therefore, it is not surprising that the different authors who have studied this evolutionary phase have obtained different results. For instance, Marigo et al. (2001) did not find any evidence of a third dredge-up in their calculations, although, as these authors point out, this effect could be due to the small number of thermal pulses followed. Chieffi et al. (2001) treated the convective boundaries according to the prescription of Herwig et al. (1997) and, moreover, extended the mixing below the convective envelope in such a way that the composition discontinuity between the helium-rich and the hydrogen-rich layers was considerably smoothed. Accordingly, these authors found a significant third dredge-up after about 13 thermal pulses. This result is similar to the one obtained by Siess et al. (2002). Unfortunately, the treatment of convection should be validated by calibrating theoretical models with observations, which is not possible for primordial stars. However, it is worth pointing out that even authors whose results for solar metallicity stars support the existence of an efficient third dredge-up (Karakas \& Lattanzio 2002) have found some evidence that might point to a less efficient third dredge-up for the case of metal-poor stars (Karakas et al. 2006). Finally, it is also important to realize that in our calculations we have not included the hot-bottom burning. In fact, it can be expected that hot-bottom burning would increase the relative abundance in the envelope of nitrogen and oxygen with respect to carbon, but not that it critically changes the total metallicity of the envelope. So, even though we have followed a relatively small number of thermal pulses (up to 20 at most), we do not expect any drastic effect on our results due to extra pollution by hot bottom-burning.

The lower left panel of Fig. 7 represents the behavior of the convective regions of the $5 M_{\odot}$ model computed with overshooting in the period of time elapsed from shortly before the beginning of the 15 th pulse until shortly after the 16 th pulse is over. In this case, the interpulse interval amounts to $2700 \mathrm{yr}$, somewhat shorter than that of the $7 M_{\odot}$ model star previously discussed. 

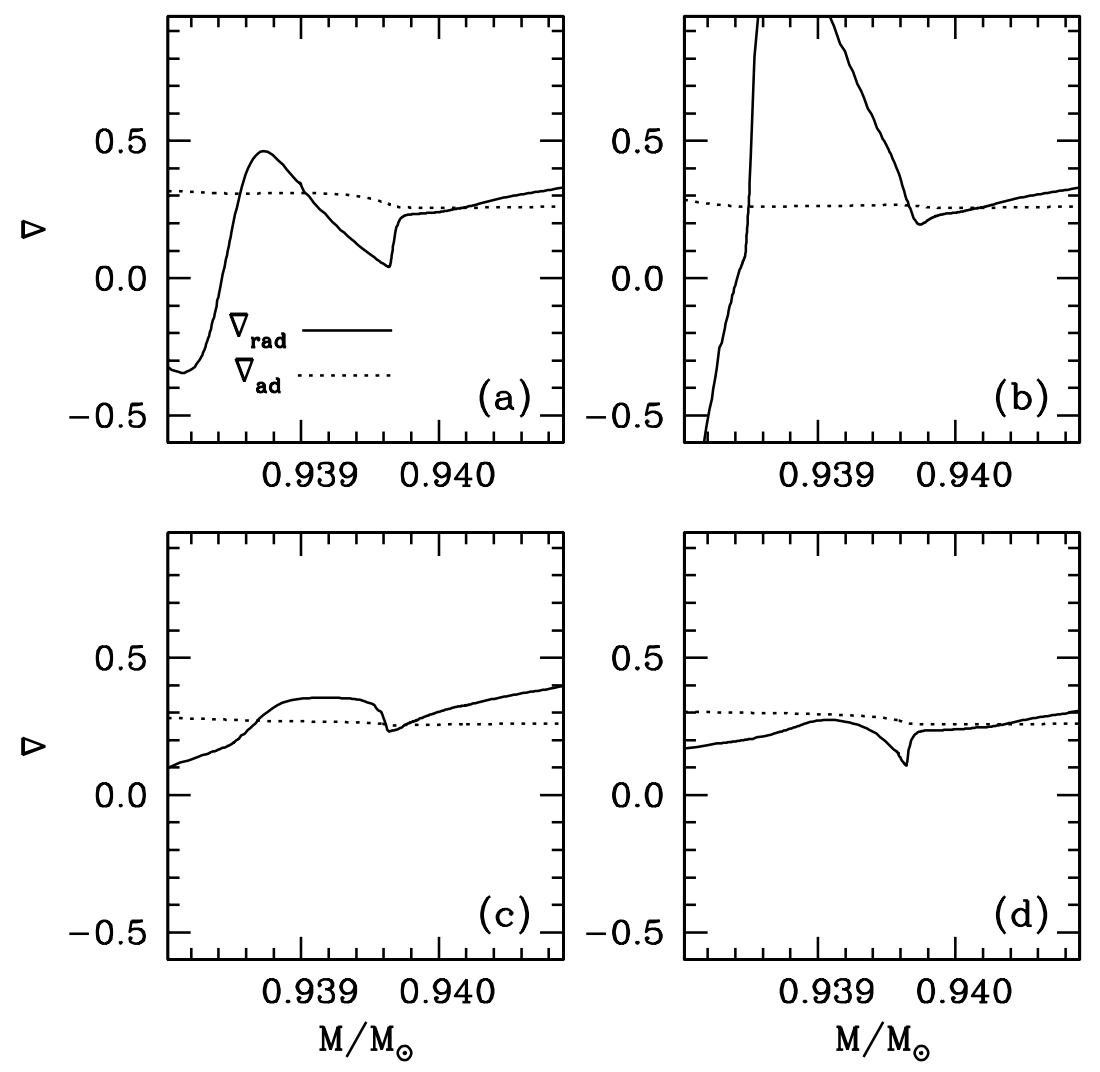

Fig. 8. Profiles of the adiabatic gradient (dotted line) and the radiative gradient (solid line) around the 20th thermal pulse of the $5 M_{\odot}$ model computed with overshooting. Panel a) shows the situation near the maximum luminosity of the pulse (at $t=2.4341617 \times 10^{15} \mathrm{~s}$ ). Panel b) depicts the same quantities at at $t=2.4341619 \times 10^{15} \mathrm{~s}$, which corresponds to the maximum extent of the inner convective zone. Panel c) shows the gradients near the maximum advance inwards of the BCE (at $t=2.4341645 \times 10^{15} \mathrm{~s}$ ). Finally, panel d) shows the gradients at the end of the pulse (at $t=2.4341699 \times 10^{15} \mathrm{~s}$ ).

Moreover, the evolution of the base of the hydrogen-rich convective envelope and of the convective zones associated with the helium flashes is totally equivalent to that already done, and the surface composition of this model does not change appreciably. The values of $\Delta M_{\mathrm{CS}}, \Delta M_{\mathrm{H}}, \Delta M_{\mathrm{DU}}$, and $\Delta M_{\mathrm{EB}}$ are also displayed in the lower left panel of Fig. 7.

To get better insight into the evolution during the occurrence of a helium flash, in Fig. 8 we have represented the adiabatic gradient $\left(\nabla_{\mathrm{ad}}\right)$, and the radiative gradient $\left(\nabla_{\mathrm{rad}}\right)$ in the region near the BCE, for some selected times during the 20th thermal pulse of the $5.0 M_{\odot}$ model sequence computed with overshooting. Panel (a) corresponds to time $t=2.4341617 \times 10^{15} \mathrm{~s}$. At this time the helium luminosity is $L_{\mathrm{He}} \simeq 2.3 \times 10^{4} L_{\odot}$ and the convective shell is just forming. Note as well that for this model the hydrogen luminosity is rather small $L_{\mathrm{H}}=4.4 L_{\odot}$ and, therefore, the BCE is able to begin its advance inwards. In the zones in which the radiative gradient is larger than the adiabatic one, according to the (modified) Schwarzschild criterion used for our calculations, there is complete mixing of the chemical elements. Panel (b) corresponds to time $t=2.4341619 \times 10^{15} \mathrm{~s}$, for which the pulse-driven convective region reaches its maximum extension $\left(\Delta M=1.36 \times 10^{-3} M_{\odot}\right)$. Panel (c) shows $\nabla_{\text {ad }}$ and $\nabla_{\text {rad }}$ at time $t=2.4341645 \times 10^{15} \mathrm{~s}$, when the BCE reaches its maximum extent inwards, at $M_{r}=0.9397 M_{\odot}$. Finally, panel (d) corresponds to time $t=2.4341699 \times 10^{15} \mathrm{~s}$ and shows the recession of the BCE once the HBS is fully operative again $\left(L_{\mathrm{H}}=1.2 \times 10^{4} L_{\odot}\right)$. As can be seen, the total amount of mass dredged-up is very small even if overshooting is taken into account.
Figure 9 shows the chemical profiles of the main nuclear species at the times already selected for panels (a) to (d) of Fig. 8. In panel (a) the ${ }^{4} \mathrm{He}$ buffer formed due to hydrogen shell burning during the previous interpulse period can be clearly seen between the mass coordinates $M_{r}=0.93900 M_{\odot}$ and $M_{r}=$ $0.93963 M_{\odot}$. Later, at time $t=2.4341619 \times 10^{15} \mathrm{~s}$, corresponding to panel (b), the effects in the chemical profile of the convective HeBS can be seen. Note the very small thickness of the region in which He has not been appreciably depleted. This small region lies between the two convective regions: the one associated with the inner He-burning shell and that associated with the H-rich envelope. In panel (b) it can be seen that the mixing due to the pulse-driven inner convective zone allows a very small enrichment in ${ }^{12} \mathrm{C}$ and ${ }^{16} \mathrm{O}$ of the regions just below the BCE. Panel (c) shows the maximum advance inwards of the BCE, which is ultimately halted by the steep composition profiles that correspond to the products of the HBS. Finally, in panel (d), one can clearly see the advance of the carbon profile, as a result of HeBS during the pulse, as well as the early recession of the BCE. In summary, even though overshooting has been taken into account, the amount of material dredged-up from the core to the convective envelope is very small.

\subsection{Models with oxygen-neon cores}

We will refer to models developing oxygen-neon cores as TP-SAGB stars. The upper and lower right panels of Fig. 6 show the first thermal pulses of the $8 M_{\odot}$ model computed without 

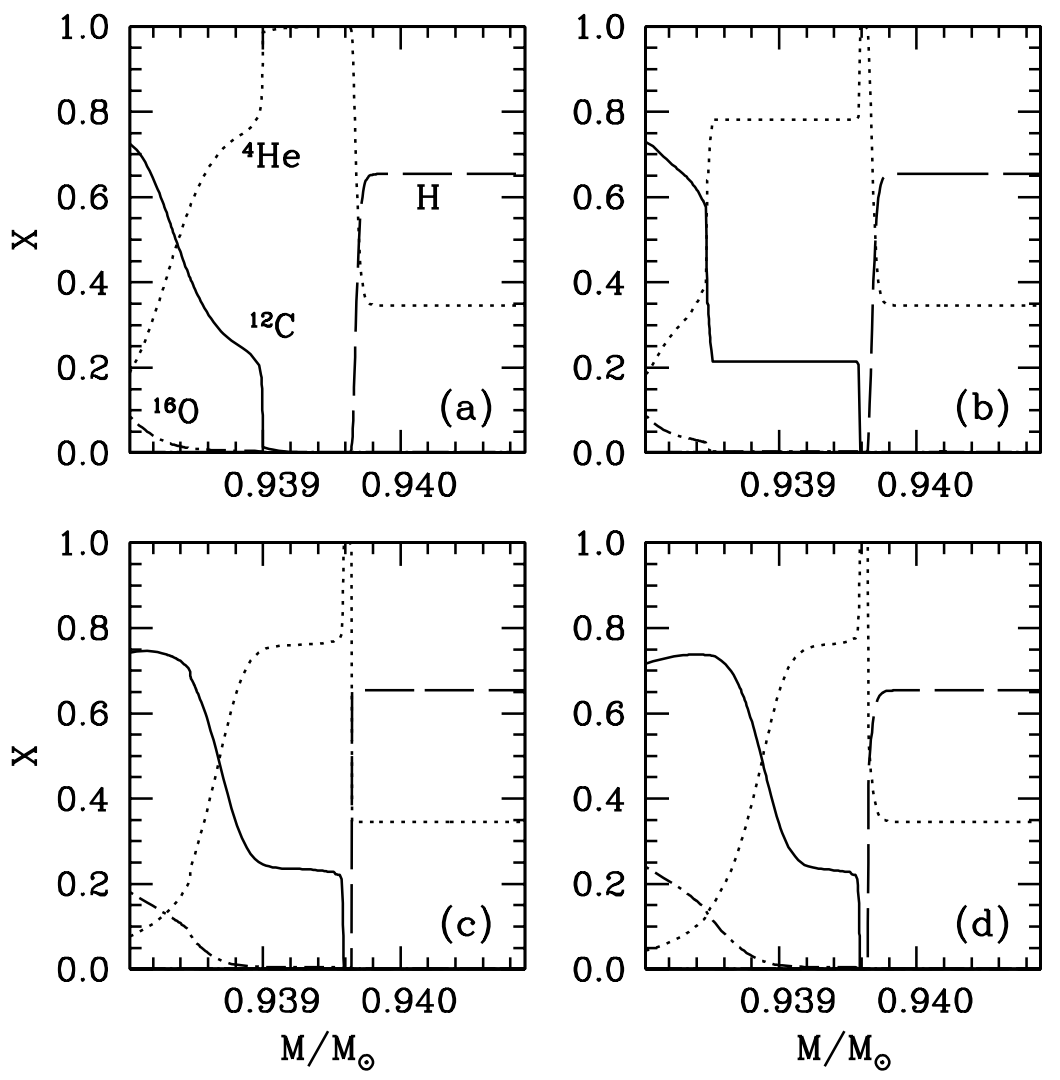

Fig. 9. Chemical profiles of the main chemical species for the $5 M_{\odot}$ model star during the 20 th thermal pulse. The evolutionary times are those of Fig. 8. The profiles are given at the maximum luminosity of the pulse (panel a)), at the maximum extent of the inner convective zone (panel b)), near the maximum advance inwards of the BCE (panel c)), and at the end of the pulse (panel d)).

Table 2. Surface abundances (by mass) of carbon, oxygen, and nitrogen at the end of our calculations for the model sequences computed without overshooting (top section) and with overshooting (bottom section).

\begin{tabular}{cccccc}
\hline \hline$M_{\text {ZAMS }} / M_{\odot}$ & $X(\mathrm{C})$ & $X(\mathrm{~N})$ & $X(\mathrm{O})$ & $\mathrm{C}: \mathrm{N}: \mathrm{O}$ & $Z$ \\
\hline 5 & $5.9 \times 10^{-9}$ & $2.0 \times 10^{-9}$ & $1.1 \times 10^{-11}$ & $1: 3.0 \times 10^{-1}: 2.0 \times 10^{-2}$ & $8.0 \times 10^{-9}$ \\
6 & $1.2 \times 10^{-7}$ & $3.1 \times 10^{-9}$ & $4.1 \times 10^{-11}$ & $1: 3.0 \times 10^{-2}: 4.0 \times 10^{-4}$ & $1.2 \times 10^{-7}$ \\
7 & $2.8 \times 10^{-6}$ & $5.7 \times 10^{-9}$ & $5.2 \times 10^{-9}$ & $1: 3.0 \times 10^{-3}: 2.0 \times 10^{-3}$ & $2.8 \times 10^{-6}$ \\
8 & $9.0 \times 10^{-5}$ & $7.4 \times 10^{-7}$ & $1.3 \times 10^{-6}$ & $1: 8.0 \times 10^{-3}: 1.0 \times 10^{-3}$ & $9.1 \times 10^{-5}$ \\
9 & $2.1 \times 10^{-4}$ & $2.0 \times 10^{-6}$ & $2.9 \times 10^{-6}$ & $1: 1.0 \times 10^{-2}: 1.5 \times 10^{-2}$ & $2.1 \times 10^{-4}$ \\
\hline 5 & $3.0 \times 10^{-7}$ & $2.0 \times 10^{-8}$ & $9.4 \times 10^{-11}$ & $1: 7.0 \times 10^{-2}: 3.0 \times 10^{-4}$ & $3.2 \times 10^{-7}$ \\
6 & $3.3 \times 10^{-5}$ & $3.8 \times 10^{-7}$ & $1.6 \times 10^{-7}$ & $1: 8.0 \times 10^{-4}: 4.0 \times 10^{-4}$ & $3.3 \times 10^{-5}$ \\
7 & $2.7 \times 10^{-4}$ & $3.7 \times 10^{-6}$ & $6.0 \times 10^{-6}$ & $1: 1.0 \times 10^{-2}: 2.0 \times 10^{-2}$ & $2.7 \times 10^{-4}$ \\
8 & $2.8 \times 10^{-3}$ & $1.4 \times 10^{-4}$ & $4.7 \times 10^{-6}$ & $1: 5.0 \times 10^{-2}: 3.0 \times 10^{-3}$ & $2.8 \times 10^{-3}$ \\
\hline
\end{tabular}

overshooting, and the corresponding ones for the $7 M_{\odot}$ model star computed with overshooting. These models have undergone extensive carbon burning and, consequently, have developed oxygen-neon cores. At the end of the carbon burning phase the core contracts while the helium burning shell is still active. As the fuel is exhausted in the innermost layers, this shell advances outward and approaches the base of the hydrogenrich convective envelope. When the distance between the latter and the HeBS is of about $10^{-4} M_{\odot}$, the base of the hydrogenrich convective envelope slightly recedes and the small radiative $\mathrm{H}$-rich region is heated. Eventually the temperature in this region reaches the critical temperature for the onset of the CNO-cycle and the HBS is reactivated. Therefore, the TP-SAGB phase develops in a very similar way to that of the models with carbonoxygen cores. The details of the pulses of the $8 M_{\odot}$ model star computed without overshooting and of the $7 M_{\odot}$ model in which overshooting was taken into account are shown in the upper and lower right panels of Fig. 7. Again, our calculations show that the enrichment in metals of the stellar surface is very small.

Table 2 lists the surface abundances (by mass) of carbon, nitrogen, and oxygen, their relative abundance ratio $(\mathrm{C}: \mathrm{N}: \mathrm{O})$, and the total metallicity $(Z)$ of the model sequences computed without overshooting (top section of Table 2) and the corresponding ones when overshooting was taken into account (bottom section). As it can be seen, the total metallicity of the surface increases with increasing $M_{\text {ZAMs }}$. The reason for this behavior is that more massive models have a lower degree of degeneracy in their cores and, therefore, the base of the convective envelope is able to penetrate deeper into the star and, consequently, is able 
to dredge-up larger amounts of material that has been processed by the different nuclear burning shells.

Another important feature of the surface abundances presented in Table 2 is that all models are carbon-rich, regardless of the adopted criterion for convection. For the models computed without overshooting (top section of Table 2) the relative abundance of nitrogen with respect to carbon first decreases, reaches a minimum at $M_{\text {ZAMS }} \simeq 7 M_{\odot}$, and then increases again. The same happens for the models computed with overshooting, but in this case the minimum is located at $M_{\text {ZAMS }} \simeq 6 M_{\odot}$. A similar behavior is obtained for the relative oxygen abundance with respect to carbon in the models computed without overshooting. For the case in which overshooting has been taken into account the relative oxygen abundance shows the reverse behavior: it first increases up to a maximum at $M_{\mathrm{ZAMS}} \simeq 7 M_{\odot}$ and then decreases. The relative abundances of nitrogen and oxygen with respect to carbon can be better understood if the models that develop carbon-oxygen or oxygen-neon cores are considered separately. For instance, the abundances of nitrogen and oxygen relative to carbon decrease as the $M_{\text {ZAMS }}$ increases for models with a carbon-oxygen core, and increase for the models that develop an oxygen-neon core.

\section{The properties of the cores}

Figure 10 shows the mass of the carbon-oxygen core after the central He burning phase for the models computed with overshooting and without overshooting as a function of the mass at the ZAMS. The mass coordinate where carbon ignition initially occurs is also shown for the models computed disregarding overshooting. Carbon ignition for the models computed with overshooting is always central for this mass range. The ZAMS mass corresponding to the intersection of the thick lines sets a lower mass limit for single $Z=0$ stars to develop ONe degenerate cores. However, our calculations show that even though this is an actual lower limit to the mass threshold for the onset of carbon ignition, the real mass threshold for the formation of an $\mathrm{ONe}$ degenerate core is about $2 M_{\odot}$ larger, as stars of masses at the ZAMS between about $5.5 M_{\odot}$ and $7.8 M_{\odot}$ do not develop carbon burning extensively enough in the carbon-oxygen core to let the core compositions be significantly changed. The vertical lines correspond to these critical masses below which carbon is burnt only partially. For the case of the models computed with overshooting, this mass threshold is located at $M_{\mathrm{ZAMS}} \simeq 6.0 M_{\odot}$, whereas for the models in which overshooting was not taken into account, the mass threshold is $M_{\mathrm{ZAMS}} \simeq 7.8 M_{\odot}$.

We have followed the evolution of all our model stars until the end of the carbon-burning phase (if this is the case) including the early stages of the thermally pulsing AGB phase. Consequently, we have been able to determine the chemical composition of the outer convective envelope and of the degenerate cores resulting from the evolution of massive intermediate mass stars, both for the case in which overshooting was considered and for the case in which it was disregarded. As will be justified in Sect. 6 with further detail - where we will discuss the possible evolutionary outcomes for this stellar mass range - the final destiny of the cores of these stars is not necessary to form single white dwarfs. This only may occur if a mechanism exists, able to produce extensive mass-loss and, hence, able to remove the convective envelope and leave behind a compact remnant. Such a mechanism could be, for instance, Roche lobe overflow due to the presence of a close companion, or, possibly, stellar winds powered by the (otherwise small) presence of metals in the envelope or, finally, by chromospherically driven

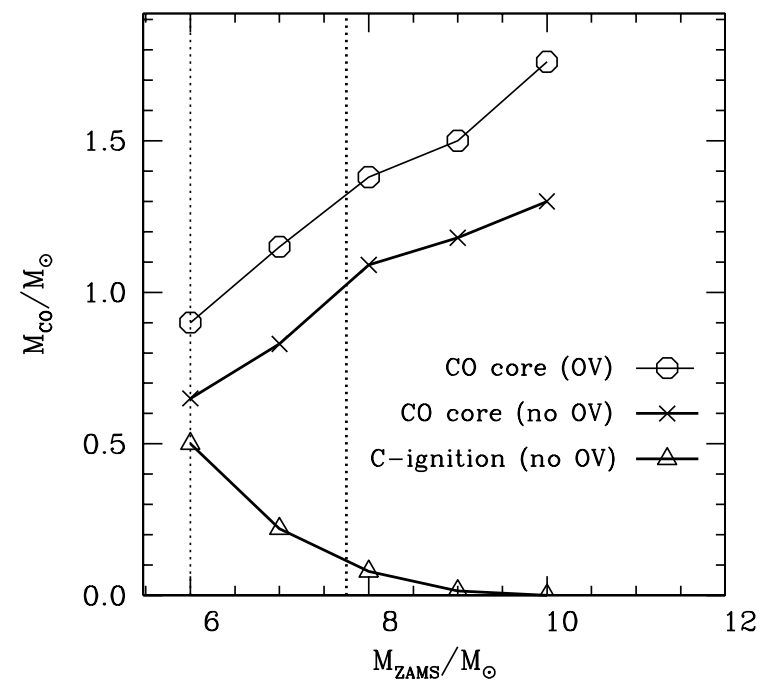

Fig. 10. Masses of the carbon-oxygen cores after the central He burning phase for the models computed without overshooting (crosses and thick line) and with overshooting (open circles and thin line). The mass coordinate for which carbon ignition occurs is also displayed for the models computed without overshooting (triangles and thick line). Finally the mass thresholds at the ZAMS for carbon burning to develop extensively are shown as vertical dotted lines.

winds (van Loon 2005). Whatever this mechanism could be - if it indeed occurs - the mass and the chemical composition of the resulting compact remnants is also a by-product of our calculations. We discuss these properties in the following subsections.

\subsection{Models computed without overshooting}

For the case in which no overshooting is adopted, primordial stars with masses at the ZAMS smaller than $7.8 M_{\odot}$ do not undergo extensive carbon burning. The pollution in metals of the stellar envelope is not large (see Table 2). Hence, radiatively driven stellar winds during this stage are not likely to be strong enough to remove the outer envelopes of these stars and produce carbon-oxygen white dwarfs, as it would be the case for solar metallicity stars of equivalent mass. Instead, the cores of these stars, due to the successive thermal pulses, could eventually grow up to the Chandrasekhar mass $\left(M_{\mathrm{Ch}}\right)$. Thus, these stars are likely to undergo a supernova explosion. This possibility will be further explored and quantified in Sect. 6. If this is indeed the case, the chemical composition of the remnant core is of interest. As an example of the resulting degenerate core, Fig. 11 shows the composition profile of the degenerate core resulting from a $7.0 M_{\odot}$, computed without overshooting.

According to our calculations, $Z=0$ stars in the range $7.8 M_{\odot} \lesssim M_{\text {ZAMS }} \lessgtr 9.0 M_{\odot}$ experience carbon burning in conditions of partial degeneracy if no overshooting is adopted. Thus, these stars form oxygen-neon cores surrounded by a carbonoxygen degenerate buffer. For this mass range we also find that the enrichment in metals of the convective envelope is rather small and, hence, is prone to undergo accretion induced collapse (AIC) also leading to a supernova explosion. The chemical composition of such cores is also relevant for the particulars of their final evolutionary phases - see, for instance, Gutiérrez et al. (1996) and Gutiérrez et al. (2005). Figure 12 shows the internal chemical profile for the case of a $M_{\text {ZAMS }}=8 M_{\odot}$ primordial star. Finally, for the case in which no overshooting is adopted, primordial stars of masses $M_{\text {ZAMS }} \gtrsim 9.5 M_{\odot}$ undergo extensive 
Table 3. Characteristics of the remnant cores at the end of our calculations for the model sequences computed without overshooting (top section), and with overshooting (bottom section).

\begin{tabular}{cccccc}
\hline \hline$M_{\mathrm{ZAMS}} / M_{\odot}$ & $M_{\mathrm{CO}} / M_{\odot}$ & $M_{\mathrm{ONe}} / M_{\odot}$ & $\Delta M_{\mathrm{CO}} / M_{\odot}$ & $X(\mathrm{C}) / X(\mathrm{O})$ & $X(\mathrm{Ne}) / X(\mathrm{O})$ \\
\hline 5 & 0.90 & - & - & 0.60 & - \\
6 & 0.92 & - & - & 0.62 & - \\
7 & 0.96 & - & - & 0.65 & - \\
8 & - & 1.01 & 0.025 & - & 0.56 \\
9 & - & 1.18 & 0.015 & - & 0.47 \\
10 & - & 1.35 & 0.010 & - & 0.35 \\
\hline 5 & 0.94 & - & - & 0.60 & - \\
6 & - & 1.00 & 0.070 & - & 0.50 \\
7 & - & 1.20 & 0.200 & - & 0.42 \\
8 & - & 1.34 & 0.002 & - & 0.36 \\
9 & - & 1.46 & 0.008 & - & 0.35 \\
\hline
\end{tabular}

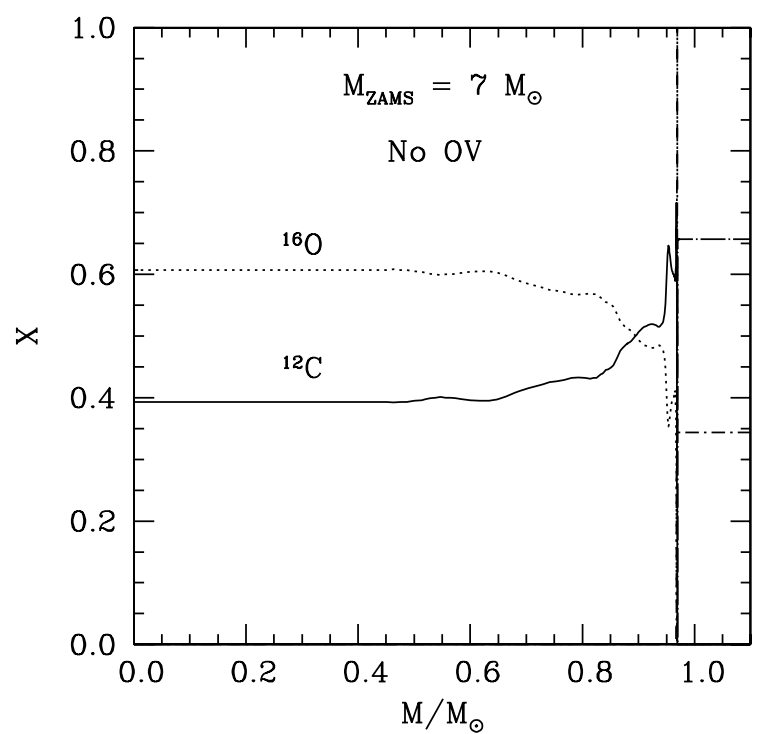

Fig. 11. Composition profile of the degenerate $\mathrm{CO}$ core for the $M_{\mathrm{ZAMS}}=$ $7 M_{\odot}$ case without overshooting.

carbon burning if no overshooting is taken into account, but the resulting oxygen-neon cores are only weakly degenerate. Such stars would most likely become SNeII directly.

The top section of Table 3 shows a summary of all this information. In particular, the mass of the $\mathrm{CO}$ core (second column) or the ONe core (third column) - depending on whether the model has avoided carbon ignition or not - at the beginning of the thermally pulsing phase are displayed. Also shown are the masses of the remaining $\mathrm{CO}$ buffers on top of the $\mathrm{ONe}$ cores $\left(\Delta M_{\mathrm{CO}}\right)$ for the models that have been able to ignite carbon (fourth column), the carbon-to-oxygen ratio in the $\mathrm{CO}$ cores of the models that fail to ignite carbon (fifth column), and the neon-to-oxygen ratio for the models that ignite carbon (last column). As can be seen, for these evolutionary sequences the mass of the carbon-oxygen buffer decreases as the mass of the ONe core increases and so does the neon-to-oxygen ratio.

\subsection{Models computed with overshooting}

The minimum mass at the ZAMS of a primordial star that allows for extensive carbon ignition and, hence, for the formation of an oxygen-neon core is, as previously mentioned, $6.0 M_{\odot}$. The convective envelopes of stars beyond this mass threshold

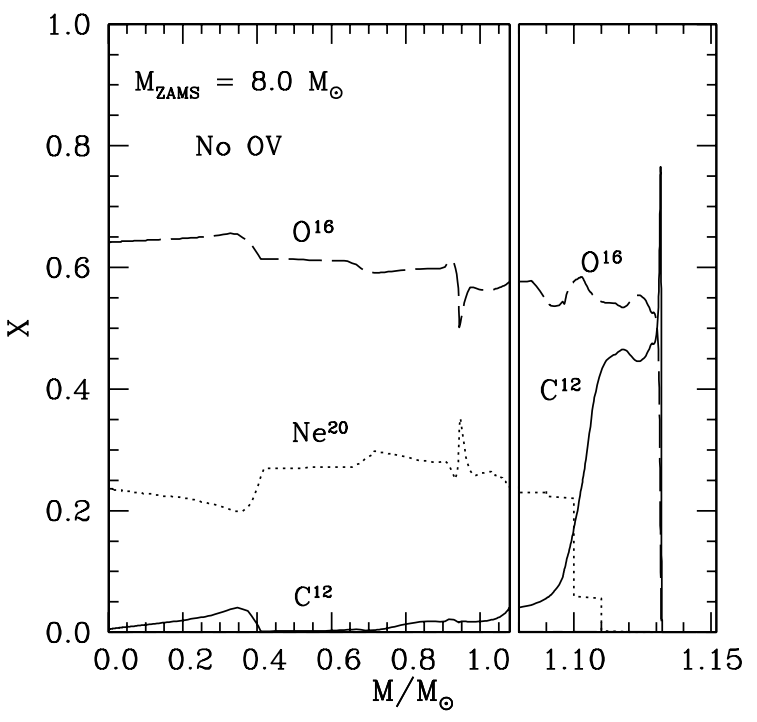

Fig. 12. Composition profile of the degenerate $\mathrm{ONe}$ core for the $M_{\text {ZAMS }}=8 M_{\odot}$ model without overshooting.

are more metal rich than those computed without overshooting. Therefore, we expect that these stars could potentially be able to support radiatively driven winds and lose their envelopes, leading to the formation of carbon-oxygen white dwarfs. In this case the core composition of these stars is also of interest because the cooling speed depends on the carbon-to-oxygen ratio (Segretain et al. 1994). Figure 13 shows an example of the resulting carbonoxygen core for the $M_{\mathrm{ZAMS}}=5 M_{\odot}$ model sequence.

Primordial stars with masses at the ZAMS between $6.5 M_{\odot}$ and $8.5 M_{\odot}$ do ignite carbon at the center and may eventually become oxygen-neon white dwarfs, provided that the convective envelope is removed by some mechanism during the thermally pulsing phase. We note however that in this case the metal content of the convective envelope is sizeable - see Table 2 - and, thus, this stars are prone to lose their convective envelopes as well. Figure 14 shows the typical final chemical profiles of such an oxygen-neon white dwarf, which do not differ substantially from those of stars of solar metallicity. Finally, for stars with masses at the ZAMS larger than $8.5 M_{\odot}$, the hydrogen-burning shell does not resurrect and, thus, these stars do not have a thermally pulsing phase. Moreover, the mass of their oxygen-neon cores is larger than $M_{\mathrm{Ch}}$ and, consequently, their evolution continues all the way to more advanced stages of stellar evolution and they will most probably produce a supernova explosion. The 


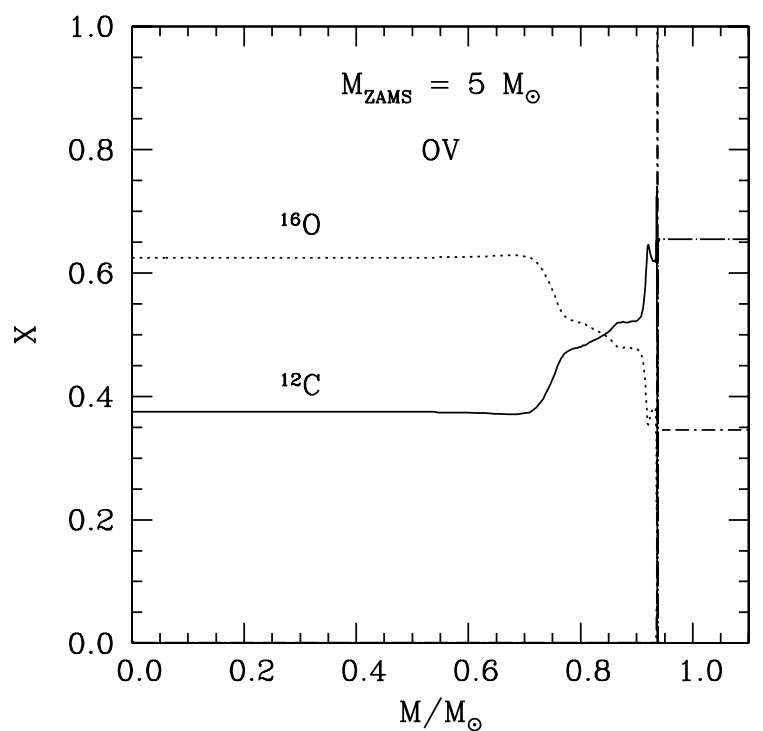

Fig. 13. Composition profile of the degenerate $\mathrm{CO}$ core for the $M_{\mathrm{ZAMS}}=$ $5 M_{\odot}$ case with overshooting.

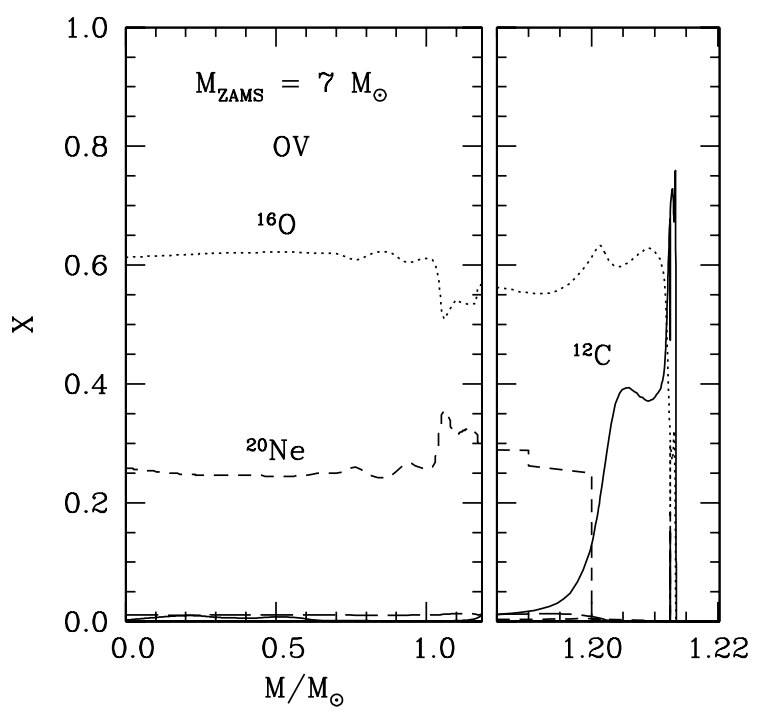

Fig. 14. Composition profile of the degenerate ONe core for the $M_{\text {ZAMS }}=7 M_{\odot}$ model with overshooting.

bottom section of Table 3 shows a summary of all this information. Finally, in Fig. 15 we present all this information graphically for both the case in which overshooting is adopted (bottom panel) or that in which it is neglected (upper panel). In both panels the total mass of the degenerate cores and the mass of the $\mathrm{ONe}$ cores are differentiated. We have also separated and labeled the regions for which at the end of our calculations we obtain a $\mathrm{CO}$ core, an ONe core, or directly a supernova. Note, however, that the final outcome of these stars still remains to be studied. This will be the goal of our next section.

\section{Mass loss and the final fate of primordial massive intermediate-mass stars}

In this section we will consider how the uncertainties that surround the evolution of primordial TP-(S)AGB stars can affect their expected final fate. We will show that, taking into account reasonable prescriptions for the mass loss rates during the TP-(S)AGB phase, the possibility that some of these stars end

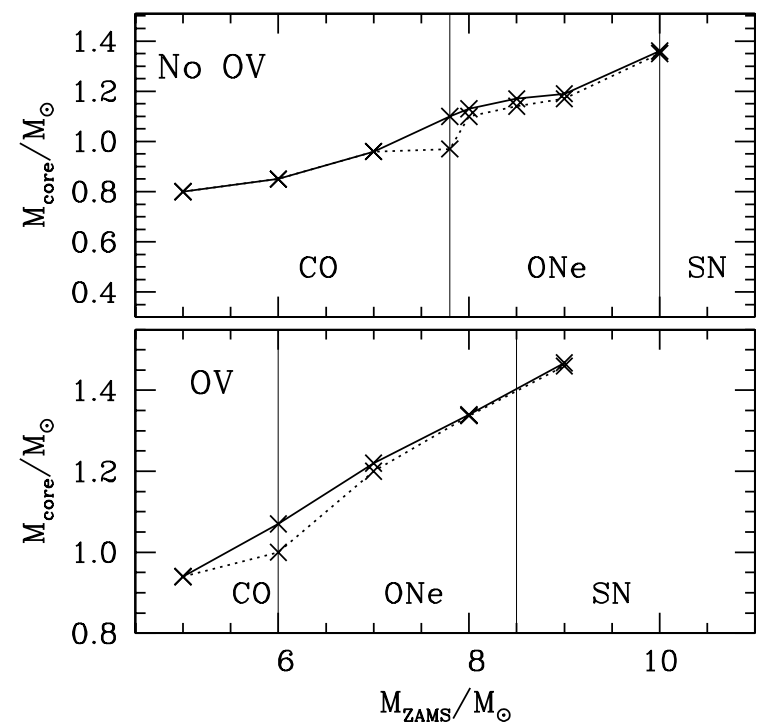

Fig. 15. Mass of the compact remnant cores by the end of our calculations for the cases with no overshooting (upper panel) and with overshooting (lower panel). The solid line shows the total mass of the degenerate core, while the dotted line is the mass of the ONe core - when applicable - without the CO buffer.

their lives as SNI1/2 - instead of white dwarfs, as is usually assumed - remains open. These supernovae are produced by the explosion of a CO core in AGB stars (Arnett 1969; Nomoto 1976). We will also show that primordial stars that form $\mathrm{ONe}$ cores near the Chandrasekhar mass will possibly undergo an electron-capture induced collapse to a neutron star, very likely producing a type II-P supernova. Our study can be considered as a first approximation to the problem, since following the full evolution of these kind of stars until the very late phases of their evolution is clearly out of our current possibilities. Synthetic (S)AGB models like those of Izzard et al. (2004) can help in this regard. Such an study is currently under way, and we postpone the discussion of such synthetic models to a forthcoming publication.

The particular details of the evolution of stars of intermediate mass from the end of the AGB phase, through a planetary nebula phase, until the formation of a white dwarf are not entirely well understood for solar metallicity stars. However, there is a general agreement on the overall picture. Strong winds are expected to remove most of the stellar envelopes before any significant growth of the cores due to shell-burning processes is achieved. Therefore, the masses of the remnant cores after the main central burning phases provide a good approximation to the final mass of the white dwarfs resulting from the evolution of these stars.

However, the uncertainties associated with the mass-loss rates due to stellar winds affect the details of the late evolutionary stages of solar metallicity stars. For the case of primordial stars, these uncertainties are more dramatic because, as it will be shown below, the adopted mass-loss rates can change the final outcome of the stellar evolutionary sequences and let them end their lives either as a white dwarf or as a supernova. In principle, the mass-loss rates are expected to be smaller for stars of low metallicity. For instance, a common prescription to take this fact into account is to adopt the following expression:

$\dot{M}(Z)=\dot{M}\left(Z_{\odot}\right)\left(\frac{Z}{Z_{\odot}}\right)^{0.5}$ 
where $\dot{M}\left(Z_{\odot}\right)$ is the mass-loss rate for solar metallicity. Note, however, that this prescription is still rather uncertain and that it is the subject of a vivid debate (van Loon 2005). However, following the recent work of Ziljstra (2004), which is suggestive of a break-down of the mass-loss rates for dust-driven winds when $Z<0.1 Z_{\odot}$, closely we adopt it as an educated guess. Other recent works that have used this expression to scale mass loss with metallicity are Kudritzki (2000), Vanbeveren (2001), and Woosley et al. (2002).

There are several prescriptions in the literature that provide $\dot{M}\left(Z_{\odot}\right)$. One may use, for instance, the expression of Schröder \& Cuntz (2005), whose authors claim it to yield reasonable results, even for the tip of the AGB:

$$
\begin{aligned}
\dot{M}_{\mathrm{SC}}\left(Z_{\odot}\right)= & \dot{M}_{\mathrm{R}}\left(Z_{\odot}\right)\left(\frac{T_{\mathrm{eff}}}{4000}\right)^{3.5} \\
& \times\left(1+\frac{g_{\odot}}{4300 \mathrm{~g}}\right) M_{\odot} \mathrm{yr}^{-1},
\end{aligned}
$$

where $T_{\text {eff }}$ is the effective temperature of the star, $g$ is its surface gravity, $g_{\odot}$ is the surface gravity of the Sun, and $\dot{M}_{\mathrm{R}}$ is the mass loss rate of Reimers (1975):

$\dot{M}_{\mathrm{R}}\left(Z_{\odot}\right)=-4 \times 10^{-13} \eta_{\mathrm{R}} \frac{L R}{M} M_{\odot} \mathrm{yr}^{-1}$,

where $\eta_{\mathrm{R}}$ is a parameter such that $1 / 3<\eta_{\mathrm{R}}<3$. Another common prescription is the expression proposed by Blöcker (1995):

$\dot{M}_{\mathrm{B}}\left(Z_{\odot}\right)=-4.83 \times 10^{-9} \frac{L^{2.7}}{M_{\mathrm{TP}}^{2.1}} \dot{M}_{\mathrm{R}}\left(Z_{\odot}\right) M_{\odot} \mathrm{yr}^{-1}$,

where $M_{\mathrm{TP}}$ is the actual mass of the considered star and, therefore, decreases as mass is lost. For solar metallicity stars this prescription yields consistently larger values during the TP-(S)AGB phase (Willson 2000; Gallart et al. 2005) as compared to the rest of the widely used prescriptions found in the literature. We adopt it because it provides an upper bound for the expected mass-loss rate.

When helium burning sets in a shell and during the thermally pulsing phase there is some dredge-up of metal-rich material and, consequently, the originally almost primordial convective envelope becomes polluted in metals. Naturally, the metallicity of the convective envelope depends on the adopted prescription for convection, and, of course, on the evolutionary model, but it is never larger than $Z \sim 2.8 \times 10^{-3}$. Taking into account Eq. (2), with the different prescriptions for $\dot{M}\left(Z_{\odot}\right)$ given by Eq. (3), with $\eta_{\mathrm{R}}=1.0$, or by Eq. (5), a rough estimate of the minimum time required to lose the envelope through radiatively driven stellar winds can be obtained:

$t_{\mathrm{env}}=\frac{\Delta M_{\mathrm{env}}}{\dot{M}}$,

where $\Delta M_{\text {env }}$ is the mass of the convective envelope. In the bottom panel of Fig. 16 we have represented the logarithm of the metallicity of the envelope, $\log Z_{\text {env }}$, at the end of our calculations for the $5,6,7,8$, and $9 M_{\odot}$ stellar evolutionary sequences computed without overshooting, and for the 5, 6, 7, and $8 M_{\odot}$ model sequences computed with overshooting. For the case of the $9 M_{\odot}$ model star computed with overshooting, we did not obtain dredge-up and, thus, the envelope was not significantly enriched in metals, leading to $Z_{\mathrm{env}}=10^{-11}$. Additionally, the degenerate core of this model star has a mass very close to the Chandrasekhar mass and, thus, the most likely outcome is a supernova. The top panel of Fig. 16 represents the corresponding mass-loss rates, as obtained by adopting the prescription of

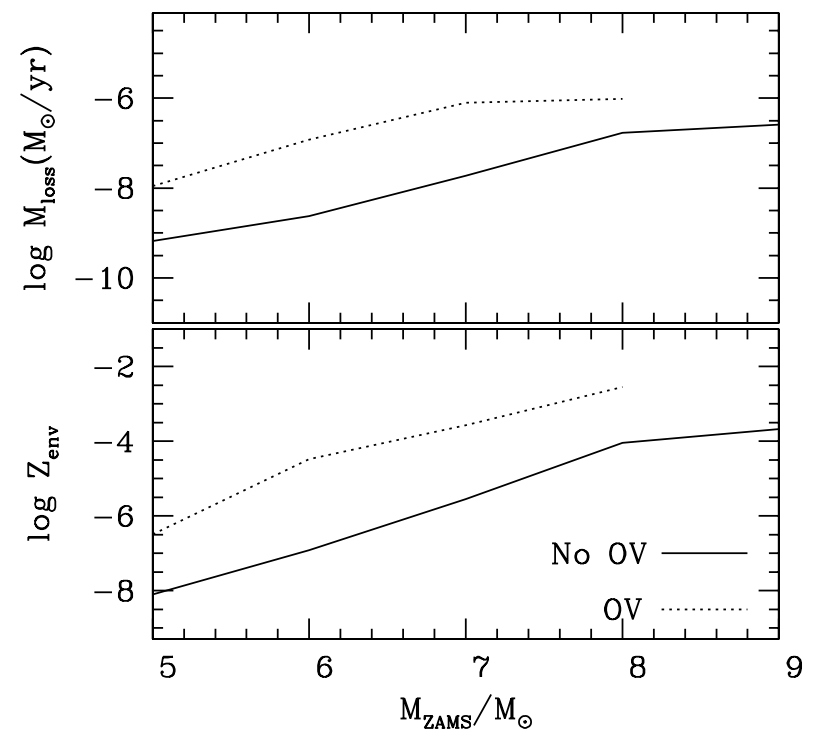

Fig. 16. Bottom panel: metallicity of the envelope of the selected models at the end of our calculations. Top panel: expected mass-loss rates given the envelope metallicities of the bottom panel when the Schröder \& Cuntz (2005) mass-loss rates are adopted. Models computed without overshooting are represented using solid lines, whereas models in which overshooting was adopted are shown with dotted lines.

Schröder \& Cuntz (2005). We remark that these mass-loss rates could be taken as a lower limit, as they have been computed taking into account the metallicity of the envelope at the end of our calculations. If the position of the BCE is, approximately, sustained and the envelope mass decreases, the freshly synthesized metals dredged-up from the core will appear less diluted in the stellar surface and hence will allow a certain increase in the mass-loss rates. On the other hand, the mass loss of primordial stars is actually hampered by the compactness and relatively high effective temperatures of these stars that result as a consequence of their low envelope metallicities. Since these stars are more compact the material lost by these stars must escape from a deeper gravitational well. Additionally, the high photospheric temperatures do not allow for the formation of dust, even if refractory elements are present. Both facts will result in smaller mass-loss rates.

Our evolutionary models yield the rate of growth of the degenerate core, $\dot{M}_{\text {core }}$, as a consequence of the successive thermal pulses and also provide us with the mass of the degenerate core. Hence, the time needed to reach the Chandrasekhar mass would be:

$t_{\text {core }} \sim \frac{M_{\mathrm{Ch}}-M_{\text {core }}}{\dot{M}_{\text {core }}}$,

for instance, for the $7 M_{\odot}$ model star computed with overshooting, the growth rate of the degenerate core during the thermally pulsing phase is $\sim 2.0 \times 10^{-7} M_{\odot} / \mathrm{yr}$. Since this model star has already an oxygen-neon core of mass $M_{\mathrm{ONe}} \simeq 1.2 M_{\odot}-$ see Table 3 - it would only need about $1.0 \times 10^{6}$ yr to reach the Chandrasekhar mass. Given the mass-loss rate shown in the top panel of Fig. 16 - about $1.7 \times 10^{-8} M_{\odot} \mathrm{yr}^{-1}-$ the time needed to get rid of the envelope would be about $3.5 \times 10^{8} \mathrm{yr}$ if the prescription of Schröder \& Cuntz (2005) is adopted for the mass-loss rate. That is, $t_{\text {env }}$ is about 350 times larger than the time needed by the star to reach $M_{\mathrm{Ch}}$. Therefore, one could reasonably expect that this star would end its life as a supernova. 
Table 4. Timescales (in years) associated with core growth and loss of the envelope for the case in which no overshooting was taken into account (top section) and for the case in which overshooting was adopted (bottom section).

\begin{tabular}{cccc}
\hline \hline$M_{\text {ZAMS }} / M_{\odot}$ & $t_{\text {core }}$ & $t_{\text {env }}^{\mathrm{SC}}$ & $t_{\text {env }}^{\mathrm{B}}$ \\
\hline 5 & $3.0 \times 10^{6}$ & $6.2 \times 10^{9}$ & $2.6 \times 10^{8}$ \\
6 & $1.9 \times 10^{6}$ & $2.1 \times 10^{9}$ & $1.2 \times 10^{8}$ \\
7 & $1.2 \times 10^{6}$ & $3.1 \times 10^{8}$ & $7.7 \times 10^{6}$ \\
8 & $1.1 \times 10^{6}$ & $4.1 \times 10^{7}$ & $6.7 \times 10^{5}$ \\
9 & $0.7 \times 10^{6}$ & $2.9 \times 10^{7}$ & $3.4 \times 10^{5}$ \\
\hline 5 & $9.0 \times 10^{6}$ & $4.1 \times 10^{8}$ & $6.6 \times 10^{6}$ \\
6 & $7.2 \times 10^{5}$ & $4.3 \times 10^{7}$ & $7.0 \times 10^{5}$ \\
7 & $5.0 \times 10^{5}$ & $6.8 \times 10^{6}$ & $4.2 \times 10^{4}$ \\
8 & $1.7 \times 10^{5}$ & $1.2 \times 10^{7}$ & $3.1 \times 10^{5}$ \\
\hline
\end{tabular}

In this respect, it must be said that our work is just a first approach, as we have assumed that the rate of core growth is constant, that the third dredge-up is going to remain inefficient during the whole TP-(S)AGB phase, and that we have not included the effects of hot-bottom burning. A more realistic treatment of the problem would require us to build up a synthetic code, as it has been done by Izzard et al. (2004), or Groenewegen \& de Jong (1994) and references therein. However, there are some facts that support the interest of our results. First of all, the fact that the times required for the growth of the core up to $M_{\mathrm{Ch}}$ are several orders of magnitude longer than the times required to get rid of the envelopes. Second, the fact that even when disregarding the effect of metallicity, we still obtain the same qualitative behavior, although the values of the times required to eject the envelope decrease considerably. Third, that using other mass loss prescriptions, such as Reimers (1975) or Vassiliadis \& Wood (1993), we also reproduce the same behavior.

The procedure explained above can be repeated for all the evolutionary sequences presented here adopting either the prescriptions of Schröder \& Cuntz (2005) or of Blöcker (1995) for the mass-loss rates. The results can be found in Table 4 . The first column of this table displays the initial masses of the models analyzed in this work, the second column shows the time needed for the core of these stars to reach the Chandrasekhar mass, $t_{\text {core }}$, as obtained from Eq. (7), and using the core growth rates obtained from our evolutionary calculations. In particular, the core growth rates have been obtained by dividing the mass growth of the core between the fifth and the last pulse computed in each model by the corresponding time interval. We have avoided the first pulses (or mini-pulses) to obtain average core growth rates during the whole thermally pulsing phase. With this procedure we obtain core growth rates ranging from $2 \times 10^{-7} M_{\odot} \mathrm{yr}^{-1}$ to $5 \times 10^{-7} M_{\odot} \mathrm{yr}^{-1}$. Because we have followed a limited number of pulses, these values could not correspond exactly to the average core growth rates during the whole thermally pulsing phase, but provide us with a reasonably good approximation for the purposes of the present work. The time needed to get rid of the envelope according to the prescription of Schröder \& Cuntz $(2005), t_{\mathrm{env}}^{\mathrm{SC}}$, is shown in the third column of Table 4 , whereas the time needed to lose the envelope according to the prescription of Blöcker (1995), $t_{\mathrm{env}}^{\mathrm{B}}$, is shown in the fourth column. As it has been done so far, the top section of Table 4 shows the results for the models computed without considering overshooting, whereas the results obtained taking overshooting into account are shown in the bottom section of this table.
If we compare the times required for these stars to lose their envelopes according to the prescription of Schröder \& Cuntz (2005) with those required by the cores to reach $M_{\mathrm{Ch}}$, it can be seen that for all the models in Table $4 t_{\mathrm{env}}^{\mathrm{SC}}$ is between one and three orders of magnitude larger than $t_{\text {core }}$. Therefore, all these stars would likely end their lives as supernovae, unless some additional mechanism enhancing the mass-loss rate played a significant role in their evolution. Rotational induced mixing could be this extra mixing mechanism required for the existence of strong stellar winds. Being completely devoid of metals, primordial stars would have no means of slowing down their initial rotation (Chiappini et al. 2006). Furthermore, because they are more compact than higher metallicity stars of similar masses, higher rotational velocities could be expected simply as a consequence of angular momentum conservation during the initial collapsing stages. Hence primordial stars might experience a substantial amount of rotational mixing (Meynet et al. 2006). It is important to point out that overshooting cannot account for this extra mixing, as rotational mixing proceeds also in radiative zones. In particular, it is important to note that Meynet et al. (2006) have followed the evolution of a $7 M_{\odot}$ star, with $Z=10^{-5}$, and have found a 1000 -fold increase in its surface metallicity. Nevertheless, it is also worth noticing that the initial rotation velocity considered in this work was near the critical rotation velocity. In any case, rotation is always difficult to introduce in a self-consistent way. Some groups assume that zero metallicity stars are fast rotators, but other advocate, on the basis of theoretical grounds, for much smaller rotation rates for primordial stars (Silk \& Langer 2006). Moreover, Yoon \& Langer (2005) point to another open question: the feedback between rotation and stellar winds. These authors conclude that the rotation rates required to allow for the mixing of the collapsar can only be kept for weak stellar winds. Consequently, this point continues to be an open issue.

The picture changes considerably when we consider the times required to lose the envelope when the prescription of Blöcker (1995) is adopted. Not surprisingly, the mass-loss rates increase and, therefore, the times required to lose the stellar envelopes decrease. The results are the following: the most massive models computed without overshooting (the 8 and $9 M_{\odot}$ model stars), and all the models computed with overshooting except the $8 M_{\odot}$ model are able to lose their envelopes before their cores reach $M_{\mathrm{Ch}}$ and, therefore, would probably end their lives as white dwarfs. The $8 M_{\odot}$ model computed with overshooting almost immediately becomes a supernova because the mass of its core $\left(1.34 M_{\odot}\right)$ is already very close to $M_{\mathrm{Ch}}$. The key factor that determines the long timescales required to lose the envelope for the models with masses between 5 and $7 M_{\odot}$ computed without overshooting is the low metallicities of the envelopes of these models. A comparison of the envelope timescales with the corresponding values of $t_{\text {core }}$ allows us to conclude that these objects are likely to end their lives as $\mathrm{SNeI} 1 / 2$, as has been previously found for all the cases computed adopting the Schröder \& Cuntz (2005) prescription for the mass-loss rates.

\section{Summary and discussion}

We have followed the evolution of massive intermediate-mass primordial stars from the zero age main sequence until the end of the carbon-burning phase and the thermally pulsing (Super)AGB phase (if this is the case). We have also studied the role of overshooting by computing two series of evolutionary sequences. In the first of these series overshooting was completely neglected. For this series of evolutionary models we followed the 
evolution of model stars with masses ranging from $5 M_{\odot}$ to $9 M_{\odot}$. In a second series of calculations overshooting was taken into account according to the prescription of Eldridge \& Tout (2004) and we followed the evolution of primordial stars with masses ranging from $5 M_{\odot}$ to $8 M_{\odot}$. This has allowed us to determine the masses of the degenerate cores resulting after the main central burning stages of primordial stars of these masses, and to study the mass limits for primordial stars to form $\mathrm{CO}$ white dwarfs, ONe white dwarfs, or to undergo a supernova explosion.

Our main results can be summarized as follows: if we consider the case in which overshooting was disregarded we have obtained a maximum mass for the formation of $\mathrm{CO}$ degenerate cores of $\sim 7.8 M_{\odot}$, and a maximum mass for the formation of an $\mathrm{ONe}$ cores of $\sim 9.5 M_{\odot}$. Stars more massive than $9.5 M_{\odot}$ form electron-degenerate cores with masses larger than the Chandrasekhar limit and, consequently, would directly undergo supernova explosion through the core collapse mechanism. If a moderate amount of overshooting is adopted, the mass threshold for the formation of a carbon-oxygen core, for the formation of a neon-oxygen core, or for a supernova explosion to be undergone is shifted to smaller masses by $\sim 2 M_{\odot}$. Therefore, we get a maximum ZAMS mass of $6.0 M_{\odot}$ for the formation of a $\mathrm{CO}$ degenerate core and a maximum value of $8.5 M_{\odot}$ for the formation of an $\mathrm{ONe}$ degenerate core. One would obtain a supernova for ZAMS masses larger than this value. For both sequences of models (those in which overshooting was taken into account and those in which it was disregarded), the resulting ONe cores appear to have a chemical stratification similar to that previously found by García-Berro et al. (1997) and Gil-Pons et al. (2005). Namely, an ONe electron-degenerate core surrounded by a relatively thick $\mathrm{CO}$ buffer whose absolute mass decreases as the mass of the degenerate $\mathrm{ONe}$ core increases. Typical masses for this $\mathrm{CO}$ buffer range from 0.04 to less than $0.01 M_{\odot}$, depending on the mass of the ONe core and on the choice of the prescription adopted for overshooting. The degeneracy in this CO buffer is also high. On top of the CO buffer there is a transition region in which helium is most abundant and the degeneracy rapidly decreases and, on top of it, a H-rich convective envelope is found.

The final outcome of these evolutionary sequences has been also analyzed in detail. Of course, this depends on both the rate of growth of the degenerate cores and on the adopted prescriptions for mass-loss. Moreover, since the mass-loss rates are most probably a function of the metallicity of the convective envelope, the final outcome of these stars depends sensitively on this value and, thus, on the possible previous dredge-up episodes. In particular, for non-primordial stars such a question would not represent a problem since stellar winds during the TP-(S)AGB stage are naturally pointed out as the mechanism by which these stars are deprived from their hydrogen-rich envelopes and, therefore, the formation of white dwarfs is immediately inferred. Such stellar winds can be easily justified by the obvious presence of metals in the envelopes of non-primordial stars. However, only the lightest elements are originally present in $Z=0$ stars. Consequently, for the case of the massive intermediate mass stars that we have analyzed in this paper the situation is not so clear. Their evolution through core hydrogen, helium and, eventually, carbon burning, and the dredge-up processes that accompany the TP-(S)AGB phase yield a modest enrichment in metals of the surface. Whether or not this enrichment is enough to enhance the mass-loss rates is unclear. For instance, we have found that in the case of the $7.0 M_{\odot}$ star computed disregarding overshooting, the surface abundance by mass of metals is of the order of $10^{-5}$ after the 15 th pulse of the TP-AGB phase, whereas in the case of the $9.0 M_{\odot}$ star, the surface abundance of metals is already of the order of $10^{-4}$ by the end of the carbonburning phase (Gil-Pons et al. 2005). The evolution during the TP-(S)AGB phase of the models computed with overshooting leads to more metal-enriched envelopes because there exists a deeper penetration of the convective envelope to the regions of the star where core elements have been synthesized and, therefore, mass loss is more favored. In spite of these metal enrichments we have shown that if the Schröder \& Cuntz (2005) prescription (corrected for the effect of the metallicity of the envelope) is adopted for the mass-loss rates during the TP-(S)AGB phase, all the models studied here seem more likely to end their lives as supernovae. The reason is that their cores are able to grow up to $M_{\mathrm{Ch}}$ in time scales between two and three orders of magnitudes shorter than those required for these stars to get rid of their envelopes, in agreement with the arguments of Zijlstra (2004).

Taking the prescription of Blöcker (1995) into account still allows the formation of SNI1/2 as a consequence of the evolution of ZAMS objects between 5 and $7 M_{\odot}$ computed without overshooting. This prescription is very often considered to produce very high mass-loss rates and, therefore, the fact that even under these conditions the possibility for the formation of SNI1/2 remains open is an encouraging result that deserves further scrutiny. In any case, it must be kept in mind that the lack of a first principle theory of mass-loss results in a considerable complication for these studies, even in the case of solar metallicity stars. The conclusions on the subject must rely on detailed observations of open clusters or detached binary systems in which one of the members is a white dwarf - see Weidemann (2000) for a thorough review - and any extrapolation to primordial objects might still somehow be speculative.

Acknowledgements. Part of this work was supported by the MCYT grant AYA05-08013-C03-01, by the European Union FEDER funds, and by the AGAUR.

\section{References}

Abia, C., Domínguez, I., Straniero, O., et al. 2001, ApJ, 557, 126 Alpher, R. A., \& Herman, R. 1950, Rev. Mod. Phys., 22, 153 Arnett, D. W. 1969, Ap\&SS, 5, 180

Bessel, M. S., \& Norris, J. 1984, ApJ, 285, 622

Blöcker, T. 1995, A\&A, 297, 727

Bowen, G. H., \& Willson, L. A. 1991, ApJ, 375, L53

Bromm, V., Coppi, P. S., \& Larson, R. B. 2002, ApJ, 564, 23

Canal, R., \& Schatzman, E. 1976, A\&A, 46, 229

Chiappini, C., Hirschi, R., Meynet, G., et al. 2006, A\&A, 449, L27

Chieffi, A., Domínguez, I., Limongi, M., \& Straniero, O. 2001, ApJ, 554, 1159

Christlieb, N., Bessell, M. S., Beers, T. C., et al. 2002, Nature, 419, 904

Demarque, P., Woo, J. H., Kim, Y. C., \& Yi, S. K. 2004, ApJS, 155, 667

Eldridge, J. J., \& Tout, C. A. 2004, MNRAS, 353, 87

Frebel, A., Aoki, W., Christlieb, N., et al. 2005, Nature, 434, 871

Gallart, C., Zoccali, M., \& Aparicio, A. 2005, ARA\&A, 43, 387

García-Berro, E., Ritossa, C., \& Iben, I. 1997, ApJ, 485, 765

Gil-Pons, P., Suda, T., Fujimoto, M. Y., \& García-Berro, E. 2005, A\&A, 433, 1037

Gorieli, S., \& Siess, L. 2001, A\&A, 378, 25

Groenewegen, M. A. T., \& de Jong, T. 1994, A\&A, 267, 410

Gutiérrez, J., Canal, R., \& García-Berro, E. 2005, A\&A, 435, 231

Gutiérrez, J., García-Berro, E., Iben, I., et al. 1996, ApJ, 459, 701

Heger, A., \& Woosley, S. E. 2002, ApJ, 567, 532

Heger, A., Woosley, S. E., \& Waters, R. 2000, in The First Stars, ed. A. Weiss, T. G. Abel, \& V. Hill (Berlin: Springer), 121

Heger, A., \& Woosley, S. E. 2002, in Proc. of the 11th Workshop on Nucl. Astrophys., ed. E. Müller, \& W. Hillebrandt, MPA Procs., Garching, 8

Herwig, F., Bloecker, T., Schoenberner, D., \& El-Eid, M. 1997, A\&A, 324, L81 
Iben, I., \& Renzini, A. 1983, ARA\&A, 21, 271

Iben, I., Ritossa, C., \& García-Berro, E. 1997, ApJ, 489, 772

Iglesias, C. A., \& Rogers, F. J. 1993, ApJ, 412, 752

Izzard, R., Tout, C., Karakas, A., \& Lattanzio, J. 2004, MNRAS, 350, 407

Johnson, J. L., \& Bromm, V. 2006, MNRAS, 366, 247

Karakas, A. I., \& Lattanzio, J. C. 2002, PASA, 19, 515

Karakas, A. I., Fenner, Y., Sills, A., et al. 2004, Mem.S.A.It., 75, 282

Kudritzki, R. P. 2000, in The First Stars, ed. A. Weiss, T. G. Abel, \& V. Hill (Berlin: Springer), 127

Machida, M. N., Tomisaka, T., Nakamura, F., \& Fujimoto, M. Y. 2005, ApJ, 622, 39

Marigo, P., Girardi, C., Chiosi, C., \& Wood, R. 2001, A\&A, 371, 152

Meynet, G., Ekström, S., \& Maeder, A. 2006, A\&A, 447, 623

Nakamura, F., \& Umemura, M. 1999, ApJ, 515, 239

Nakamura, F., \& Umemura, M. 2001, ApJ, 548, 19

Nomoto, K., Sugimoto, D., \& Neo, S. 1976, Ap\&SS, 39, 37

Olive, K. A. 2000, Nucl. Phys. Proc. Suppl., 80, 79

Reimers, D. 1975, Mem. Soc. R. Sci. Liège, Ser. 6, 8, 369
Ritossa, C., García-Berro, E., \& Iben, I. 1995, ApJ, 460, 489

Ritossa, C., García-Berro, E., \& Iben, I. 1999, ApJ, 515, 381

Schneider, R., Salvaterra, R., Ferrara, A., \& Ciardi, B. 2005, MNRAS, 369, 825

Schröder, K. P., Pols, O. R., \& Eggleton, P. P. 1997, MNRAS, 285, 696

Schröder, K. P., \& Cuntz, M. 2005, ApJ, 630, L73

Segretain, L., Chabrier, G., Hernanz, M., et al. 1994, ApJ, 434, 641

Siess, L., Livio, M., \& Lattanzio, J. 2002, ApJ, 2002, 570

Vanbeveren, D. 2001, in The Influence of Binaries on Stellar Population Studies, ed. D. Vanbeveren, Astrophysics and Space Science Library No. 264 (Dordrecht: Kluwer), 1

van Loon, J. T., Cioni, M. R. L., Zijlstra, A. A., \& Loup, C. 2005, A\&A, 438, 273

Vassiliadis, E., \& Wood, P. R. 1993, ApJ, 413, 641

Willson, L. A. 2000, ARA\&A, 38, 573

Woosley, S., Heger, A., \& Weaver, T. 2002, Rev. Mod. Phys., 43, 1015

Yoon, S. C., Langer, N., \& Scheithauer, S. 2004, A\&A, 425, 207

Young, P., Knierman, K. A., Rigby, J. R., \& Arnett, D. 2003, ApJ, 595, 1114

Zijlstra, A. A. 2004, MNRAS, 348, L23 